\title{
Forecasting U.S. Tourist Arrivals using Singular Spectrum Analysis
}

April 8, 2014

\begin{abstract}
This paper introduces Singular Spectrum Analysis (SSA) for tourism demand forecasting via an application into total monthly U.S. Tourist arrivals from 1996-2012. The global tourism industry is today, a key driver of foreign exchange inflows to an economy. Here, we compare the forecasting results from SSA with those from ARIMA, Exponential Smoothing (ETS) and Neural Networks (NN). We find statistically significant evidence proving that the SSA model outperforms the optimal ARIMA, ETS and NN models at forecasting total U.S. Tourist arrivals. The study also finds SSA outperforming ARIMA at forecasting U.S. Tourist arrivals by country of origin with statistically significant results. In the process, we find strong evidence to justify the discontinuation of employing ARIMA, ETS and a feedforward NN model with one hidden layer as a forecasting technique for U.S. Tourist arrivals in the future, and introduce SSA as its highly lucrative replacement.
\end{abstract}

Keywords: United States; Tourist arrivals; Tourism demand; Forecasting; Singular Spectrum Analysis; ARIMA; Exponential Smoothing; Neural Networks.

\section{Introduction}

Previous research has highlighted the importance of accurate demand forecasting to the tourism sector. The dependence of tourism on both investment and infrastructure development make a degree of advance planning essential, as many authors have recognised. Well informed investment decisions are vital for efficient resource allocation in both tourism and supporting sectors. The economic downturn and an increased awareness of world economic volatility have strengthened rather than weakened this need to forecast tourist demand accurately.

As discussed in the following section there is an extensive and high profile existing literature on forecasting tourism demand. This literature covers a wide range of different forecasting techniques, applied to a wide range of different countries or locations. The purpose of this paper is to add to this literature by introducing a new model for forecasting tourist arrivals and to apply it to inbound U.S. Tourist arrivals. Forecasting U.S. Tourist arrivals is both a demanding and important task, mainly because these data exhibit a high degree of fluctuation over time. Figure 1 depicts the time series for total monthly U.S. Tourist arrivals between January 1996 and November 2012. A first look at the time series suggests signs of seasonality in U.S tourist arrivals. The figure also shows that the tourism industry in the U.S. is experiencing rapid development in terms of demand. Since 2002 U.S. Tourist arrivals exhibit a strong upward trend. The need to 
allocate resources for future growth is further evidence of the importance of developing accurate demand forecasting for investors, managers and policy makers in the tourism sector.

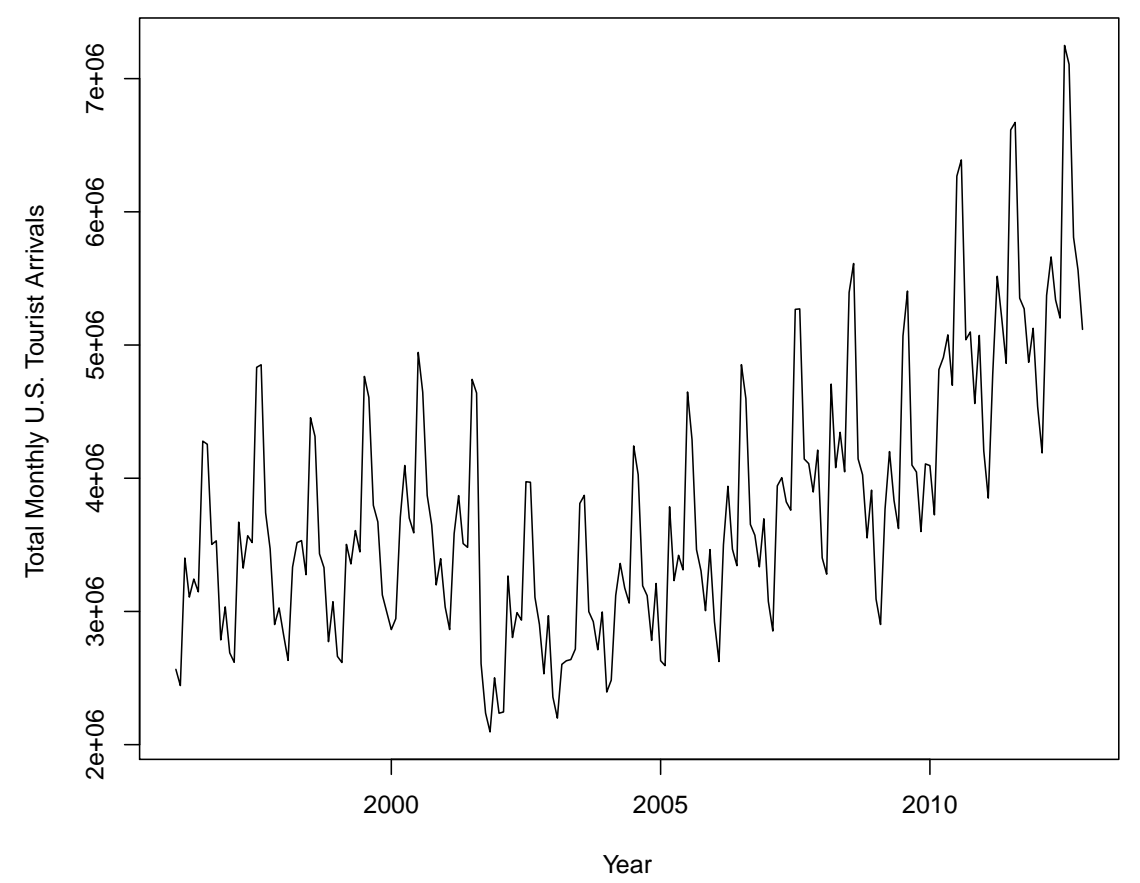

Figure 1: Total monthly U.S. Tourist arrivals time series (Jan. 1996 - Nov. 2012).

There are a number of components which define a good demand forecasting model for tourism management. Firstly, the forecasting model has to be able to pick up strong variations in tourist arrivals as most tourist demand time series show increasing fluctuations with seasons. Secondly, given the seasonal fluctuations, the measure of forecasting accuracy based on the forecasting error alone is not sufficient. It is important that the forecasting model is equally able to predict the actual direction of change. If not, investment decisions and the resources allocated to tourism could find themselves catering for a peak in demand but actually experiencing a trough. Thirdly, a tourism demand forecasting model needs to be efficient both in the short and long run. This is because long term investments are needed to be able to supply to the short term demand fluctuations. In this paper we consider all these aspects as we introduce the Singular Spectrum Analysis (SSA) technique for forecasting U.S. Tourist arrivals and compare its performance with other forecasting models currently used to forecast tourism demand. In brief, the SSA technique seeks to decompose the original time series, filter the noise and reconstruct a new time series which is less noisy. We then use this newly reconstructed time series for forecasting future data points.

The remainder of this paper is organized as follows. Section 2 provides a review of the existing literature on the forecasting of tourism demand. Section 3 provides a review of the main forecasting techniques employed. Section 4 introduces the data for U.S. inbound tourist arrivals and discusses the measures for evaluating the forecasting performance. Section 5 reports the empirical results from the SSA technique in comparison to other, previously employed, forecasting techniques. Conclusions are provided in Section 6. 


\section{Literature Review}

The existing literature on the forecasting of tourism demand is wide ranging both in terms of the different techniques employed and in terms of the different countries covered. A common theme in almost all of the papers also helps to explain the reasons behind this extensive interest in forecasting tourism demand. A large number of authors including Chan and Lim (2011), Chu (2008), Coshall and Charlesworth (2011) and, Goh and Law (2002) emphasise the importance of forecasting for investment and development planning in tourism. This message is re-enforced by authors, such as Gounopoulos, Petmezas, and Santamaria (2012) and Hui and Yuen (2002), who add that such forecasts are also important as a consequence of the vulnerability of tourism to large fluctuations in demand. Some authors also emphasise the importance of tourism to a particular economy to re-enforce the importance of accurately forecasting tourism demand. Examples include work by Jackman and Greenidge (2010) for Barbados and Chu (2011) for Macau. Those readers seeking a detailed review of the literature, the paper by Song and Li (2008) covers 121 studies produced from 2000 to the date of publication. This review article offers a further reason for the sustained and extensive interest in forecasting tourism demand. They found that no single forecasting model outperforms all other in all possible situations. This implies that the literature is not only of importance but also in need of further research. A more recent review of forecasting and the closely related issue of tourism demand modelling is included in the paper by Song et al. (2012).

Perhaps the most common form of study is one that assesses the performance of one or more forecast techniques relative to a set of alternatives. Alvarez-Diaz and Rossello-Nadal (2010) examine forecasts of UK tourist arrivals in the Balerics, using meteorological variables. They compare the performance of an ARIMA model and a non-causal autoregressive neural network, finding that the latter performs better. Assaf, Barros, and Gil-Alana (2011) examine persistence and seasonality in data for tourist arrivals into Australia. They compare the performance of three different forecasting models, two standard methods using stationarity of degrees 0 and 1 and a model with fractional degrees of integration. Athanasopoulos and De Silva (2012), in a study of tourist arrivals in Australia and New Zealand propose a model which captures time varying seasonality within a vector innovation time series model. They produce evidence that this model offers greater forecast accuracy than a number of alternatives. Cho (2003) investigates three different techniques (exponential smoothing, univariate ARIMA and artificial neural networks) to forecast tourist arrivals in Hong Kong, finding the artificial neural networks forecasts to be the most accurate.

Chu (2008) explores fractionally integrated ARMA models in forecasting tourism arrivals in Singapore, observing that they perform well in comparison to more traditional ARIMA models. Chu (2011) compares a piecewise linear model with autoregressive trend, seasonal ARIMA and fractionally integrated ARMA models in forecasting tourism demand for Macau, concluding the piecewise linear model to be the most accurate. Likewise, Gil-Alana (2005) considers forecasts using monthly data for tourist arrivals into the US using a procedure combining unit and fractional integration in seasonal variation. He finds evidence of long memory and mean reverting behaviour. Goh and Law (2002) use data for Hong Kong tourist arrivals to compare forecasts from a stochastic non-stationary seasonality model (SARIMA) and an intervention component model (MARIMA) with a selection of eight other time series models. Their results suggest the SARIMA and MARIMA models to have the highest forecast accuracy of the models analysed.

Greenidge (2001) uses a structural time series model to provide and evaluate forecasts for tourism arrivals in Barbados. Jackman and Greenidge (2010) further explore the structural time series model for tourist arrivals in Barbados, finding that it produces more accurate forecasts 
than a number of alternatives. Hadavandi et al. (2011) present forecasts for tourism arrivals in Taiwan using a hybrid artificial intelligence model, involving a fuzzy rule-based system, which they found to be more accurate than a selection of three alternative approaches. Kim et al. (2011) consider the performance of prediction intervals for tourism arrivals into Hong Kong and Australia for a selection of time series forecasting models. They find an autoregressive bias corrected bootstrap model to perform best of those tested. Lim and McAleer (2001) analysed the performance of various different exponential smoothing models in forecasting tourist arrivals in Australia, concluding that using models expressed in first differences increased forecast accuracy. Shareef and McAleer (2007) evaluate the abilities of ARMA models to capture the effects of volatility in the time series of tourism arrivals in the Maldives. Song et al. (2010) focus on a different aspect of forecasting tourism demand - what is the appropriate measure of demand? Using data for Hong Kong they find use of tourism arrivals to be more affected by income in the country of origin and tourism expenditure to be more sensitive to prices. Wan, Wang, and Woo (2013), also using tourist arrival data for Hong Kong, assess the properties of disaggregated forecasts using a seasonal ARIMA model relative to aggregate forecasts. They find the sum of disaggregated forecasts to provide greater accuracy than an aggregate forecast.

A very closely related strand in the literature seeks to combine two or more forecasting models into a new hybrid model and to test whether this results in greater forecast accuracy. Andrawis, Atiya, and El-Shishiny (2011) finds that, in forecasts of tourism arrivals into Egypt, combining short and long term forecasts improves accuracy compared to the individual forecasts. Cang (2011) examines tourism arrivals into the U.K. and examines three different forecasting models support vector neural networks, seasonal ARIMA and an exponential smoothing model. He finds that non-linear combinations of these models offer greater forecast accuracy than the individual specifications. Coshall and Charlesworth (2011) consider a number of forecasting models, both individually and in combination. Using data on UK outbound tourism they also find that forecast accuracy is improved by using a combination of forecasts.

Shen, Li, and Song (2008) focus on outward leisure tourism from the U.S. and examine seven different types of individual forecasting techniques. Their results also suggest that forecast accuracy is improved by combining forecasts. Shen, Li, and Song (2011) conduct a similar analysis of UK outward tourism, using seven different individual forecasting methods and six combinations. Again their findings suggest that forecast accuracy is improved by using combinations of forecasts. Song et al. (2011) develop a model to forecast Hong Kong tourist arrivals which combines a structural time series model with a time varying parameter one. They find that, relative to a number of time series models, their hybrid model exhibits greater forecast accuracy. Song, Gao, and Lin (2013), again with respect to tourism arrivals in Hong Kong, consider a model which combines quantitative forecasts which judgemental forecasting from an online survey. They find that adding a judgemental component improves forecast accuracy.

A number of papers consider the implications of shocks to one or more forecasting models of tourism demand. Gounopoulos, Petmezas, and Santamaria (2012) consider the forecasting of the impact on tourism arrivals in Greece of macro-economic shocks. They compare a number of different forecasting methods, finding an ARIMA model to be the most accurate and also develop a VAR model. Smeral (2010) examines the effects on forecasts of outbound travel of global recession for a sample of countries. Mao, Ding, and Lee (2010) use a cusp catastrophe model to forecast the rates of recovery of tourist arrivals in Taiwan from the SARS epidemic. Their results suggest that tourism from China and the U.S. recovered quickly but that from Japan did not. In a similar vein Page, Song, and Wu (2012) estimate the negative effect of the Swine flu epidemic on U.K. tourist arrivals using a time varying parameter model. Fourie and Santana-Gallego (2011) use a gravity model to estimate and predict the impact of mega-sports 
tourism events on tourist arrivals.

Studies which examine the determinants of demand for tourism are not analysis of forecasting models but are so closely related to the forecasting of tourism demand that they merit consideration. Chan and Lim (2011) analyse seasonality in New Zealand tourism demand using spectral analysis. They find different categories of inbound tourism share common cyclical behaviour. Naude and Saayman (2005) consider the determinants of tourist arrivals in 43 African countries, finding tourism infrastructure and health risks to be of particular importance. Nelson et al. (2011) estimate a demand model for visitors to Hawaii from mainland U.S. Their results suggest home state income, airfares and $(\log )$ distance to be important. Seetanah, Durbarry, and Ragodoo (2010) estimate tourism demand for South Africa using a gravity model. Their analysis suggests prices, level of development and common borders to all be important determinants. Seetaram (2010) uses dynamic panel cointegration to estimate demand elasticities for tourism arrivals into Australia, finding demand to be inelastic in the short run but elastic in the long run.

Volatility models are built upon an ARIMA model to which they add a second equation to explain the conditional variance. Coshall (2009) provides a good overview of these techniques and their application to forecasting tourism demand. The most commonly used specification is the GARCH model, developed by Bollesrslev (1986). This adds to the ARIMA model an equation to explain the conditional variance. This equation models the current period conditional variance in terms of lagged squared residuals (capturing the short run impact of past shocks) and longer term effects from lagged values of the conditional variance. Extensions of the GARCH model include the TGARCH (which use dummy variables to model asymmetric shocks) and EGARCH models. For example, Kim and Wong (2006) use both the EGARCH and TGARCH models to provide forecasts of tourism demand in Korea with asymmetric responses to news shocks. Coshall (2009), in an application to UK outbound tourism, shows that forecasts using the EGARCH model can be combined with those from an exponential smoothing model such that the combined forecast is more accurate than either of the individual methods.

The use of Singular Spectrum Analysis (SSA) in the tourism sector was firstly evaluated by Beneki et al. (2012) via an application into signal extraction and forecasting of U.K. Tourist income. Here, we introduce SSA as a new model for forecasting tourism demand in the future. The SSA technique is swiftly being recognized as a powerful, nonparametric time series analysis and forecasting technique. The roots of SSA are closely associated with Broomhead and King (1986a, 1986b). The applications of SSA are diverse and its growing success is evident in many different fields (see for example, Lisi \& Medio, 1997; Ghil et al. 2002; Hassani, Heravi, \& Zhigljavsky, 2009; Ghodsi et al. 2009; Hassani \& Thomakos, 2010; Hassani, Heravi, \& Zhigljavsky, 2012; Hassani, Soofi, \& Zhigljavsky, 2013; Beneki \& Silva, 2013; Silva, 2013). As noted above, there exists various different techniques which have been applied for forecasting tourism demand in the past. In this paper we compare the forecasting results from SSA with those obtained from ARIMA (Automatic-ARIMA), Exponential Smoothing (ETS) and Neural Networks (nnetar). The ETS methodology gained its popularity through the sound performance at the M3-competition, and the state space framework which now underlies the newly developed ETS is widely applicable, like ARIMA, and provides a forecast with the backing of a good stochastic model (Hyndman et al. 2002). Neural networks has frequently been adopted in tourism demand forecasting as mentioned earlier on. It is important to note that here we use the most basic version of Vector SSA along with optimal choices, and the optimal version of ARIMA. Given the choice of forecasting methods we are comparing the forecasting accuracy provided by both parametric and nonparametric time series analysis and forecasting techniques. In comparison to parametric forecasting techniques, nonparametric techniques are not bound by 
any of the parametric assumptions such as stationarity and normality. As such, nonparametric models are able to provide a true approximation of the real situation. Also it is not the intention of this paper to show SSA as the universally best model for forecasting tourist arrivals. Instead, we are interested in introducing SSA as an alternative method, and further research is required to compare SSA's performance against many other forecasting techniques.

\section{Forecasting Methods}

\subsection{Auto-Regressive Integrated Moving Average (ARIMA)}

We employ the most optimal version of Box and Jenkins (1970) ARIMA model. This optimal version of ARIMA is provided via the forecast package in the $\mathrm{R}$ software and is more popular as Automatic-ARIMA. This particular version was mainly developed to enable ease of use, and provide swift, optimal forecasting results for those adopting the ARIMA method. A detailed description of the algorithm and the optimality of Automatic-ARIMA can be found in Hyndman and Khandakar (2008). The Automatic-ARIMA algorithm is summarized below. In doing so we mainly follow Hyndman and Athanasopoulos (2013).

In terms of determining the number of differences $d$, required for the ARIMA modelling process, the algorithm allows one to select this value using three different approaches; KPSS unit root tests, Augmented Dickey-Fuller (ADF) test or the Phillips-Perron (PP) unit root tests. It has been found that the KPSS tests lead to better forecasts in comparison to the ADF and PP test when applied to the M3 competition data (Hyndman 2014). However, instead of relying on these results alone, in this paper we consider modelling using all three approaches and report the results based on the KPSS unit root tests from Kwiatkowski et al. (1992) for the number of differences $d$ required as it provided better forecasts for U.S. Tourist arrivals in comparison to ADF and PP tests. It should also be noted that according to Hyndman and Athanasopoulos (2013), when a time series is nonstationary, the Automatic-ARIMA forecasting algorithm accounts for this by automatically taking first differences of the data until the data are stationary.

Thereafter, $p$ and $q$ are calculated by minimizing the Akaike Information Criterion (AIC). The optimal model will then be the model with the smallest AIC, and it is selected from $\operatorname{ARIMA}(2, d, 2), \operatorname{ARIMA}(0, d, 0), \operatorname{ARIMA}(1, d, 0)$ and $\operatorname{ARIMA}(0, d, 1)$. Finally, the constant $c$ is included or set to zero in the model depending on whether $d=0$ or whether $d \geq 1$. Log transformations can be attained by setting lambda $=0$ where necessary to comply with the parametric restrictions underlying ARIMA. Table 2 shows the ARIMA model parameters used for forecasting total U.S. Tourist arrivals whilst the model parameters for forecasting U.S. Tourist arrivals by country of origin are shown in Table 7 .

\subsection{Exponential Smoothing (ETS)}

The ETS technique is an automatic forecasting model incorporating the foundations of exponential smoothing and provided through the forecast package for the R software. Earlier models of exponential smoothing did not provide a method for easily calculating prediction intervals (Makridakis, Wheelwright, \& Hyndman 1998). For a detailed description of ETS refer to Hyndman and Athanasopoulos (2013). In brief, the ETS model considers the error, trend and seasonal components in choosing the best exponential smoothing model from over 30 possible options by optimizing initial values and parameters using the MLE for example and selecting the best model based on the AIC. As noted in Hyndman and Athanasopoulos (2013), both multiplicative 
and additive models give the same point forecasts with varying prediction intervals. Here we report the most favourable results for ETS by evaluating between point forecasts and prediction intervals. The ETS model parameters for forecasting total U.S. Tourist arrivals are reported in Table 3.

\subsection{Neural Networks (NN)}

Neural networks is a popular forecasting method for tourism demand according to the literature review in Section 2. Here we use an automatic forecasting model known as nnetar and provided through the forecast package in R. For a detailed explanation on how the nnetar model operates, see Hyndman et al. (2013). In brief, nnetar is structured as a system of feed-forward neural networks with lagged inputs and one hidden layer. The nnetar function trains 25 networks by using random starting values and then obtains the average of the resulting predictions to compute the forecasts. It should be noted that the simplest form of NN models contain no hidden layers and are then equivalent to linear regression. One of the NN model parameters are referred to as 'weights' and these are selected based on a loss function embedded into the learning algorithm. This loss function could for example be the Mean Squared Error (MSE) or even the Root Mean Squared Error (RMSE) which has been adopted for the loss function in the SSA algorithm explained below. We leave the automatic NN forecasting model to select the best parameters to suit the data. The resulting model parameters for NN model are shown in Table 3.

\subsection{Singular Spectrum Analysis (SSA)}

The SSA technique is different and advantageous in comparison to classical time series methods as the techniques which fall under the latter, forecasts both the signal and noise (assuming that a time series consists of signal and noise) whilst SSA seeks to filter the noise and forecast the signal. The univariate SSA technique has two variations known as Vector SSA (VSSA) and Recurrent SSA (RSSA). In this paper we concentrate on the application of the VSSA model for forecasting U.S. Tourist arrivals. The choice of VSSA over RSSA is motivated by two factors. First and foremost, the total U.S. Tourist arrivals time series (see, Figure 1) shows the presence of shocks around the periods of 2002 and 2008. Golyandina, Nekrutkin, and Zhigljavsky (2001) found the VSSA model is more robust in comparison to the RSSA model, and thus able to provide better forecasts in the presence of such shocks which create structural breaks in a time series. This was later confirmed by Pepelyshev (2010) who also provides a detailed comparison between recurrent and vector forecasting. Secondly, instead of solely relying on Golyandina, Nekrutkin, and Zhigljavsky's (2001) and Pepelyshev (2010) findings, we compared the performance of both VSSA and RSSA models at forecasting total U.S. Tourist arrivals (results are not reported here) and found no statistically significant difference in the forecasting accuracy between the two methods, except at $h=12$ steps ahead where the VSSA model outperformed the RSSA model with statistically significant results. Thus, taking these two factors into consideration, we selected the VSSA model as the more suitable counterpart for forecasting U.S. Tourist arrivals. We use the R software to obtain the VSSA forecasts with the aid of an automatic VSSA forecasting code. In brief, the SSA technique can be summarised as follows. The SSA technique has two stages and two choices as mentioned in Hassani and Mahmouvdvand (2013). The two stages are referred to as Decomposition and Reconstruction, whilst the two choices are known as the window length $L$ and the number of eigenvalues $r$. Each of the two stages include two separate steps known as Embedding, Singular Value Decomposition (SVD) and, Grouping and Diagonal Averaging. A detailed description on the theoretical and 
practical foundations of SSA can be found in Golyandina, Nekrutkin, and Zhigljavsky (2001) and Hassani (2007) which explains the process with examples. Here we provide a brief summary of the basic SSA process, and in doing so we mainly follow Hassani (2007).

\section{Stage 1: Decomposition}

\section{1st step: Embedding}

The first step is concerned with mapping a one dimensional time series $Y_{N}=\left(y_{1}, \ldots, y_{N}\right)$ into the multi-dimensional series $X_{1}, \ldots, X_{K}$ with vectors $X_{i}=\left(y_{i}, \ldots, y_{i+L-1}\right)^{\prime} \in \mathbf{R}^{L}$, where $K=N-L+1$. This process is referred to as embedding whilst the vectors $X_{i}$ are called $L$-lagged vectors. The single choice of the embedding stage is the window length $L$, which is an integer such that $2 \leq L \leq N$. This step results in the trajectory matrix $\mathbf{X}$, which is also a Hankel matrix and takes the form: $\mathbf{X}=\left[X_{1}, \ldots, X_{K}\right]=\left(x_{i j}\right)_{i, j=1}^{L, K}$.

\section{2nd step: Singular Value Decomposition (SVD)}

Next we obtain the singular value decomposition of the trajectory matrix and represent it as a sum of rank-one bi-orthogonal elementary matrices. The eigenvalues of $\mathbf{X X}^{\prime}$ are denoted by $\lambda_{1}, \ldots, \lambda_{L}$ in decreasing order of magnitude $\left(\lambda_{1} \geq \ldots \lambda_{L} \geq 0\right)$ and by $U_{1}, \ldots, U_{L}$ the orthonormal system (that is, $\left(U_{i}, U_{j}\right)=0$ for $i \neq j$ and $\left\|U_{i}\right\|=1$ of the eigenvectors of the matrix $\mathbf{X X}^{\prime}$ corresponding to these eigenvalues. Here, $\left\|U_{i}\right\|$ is the norm of the vector $U_{i}$, and $\left(U_{i}, U_{j}\right)$ is the inner product of the vectors $U_{i}$ and $U_{j}$. Set

$$
d=\max \left(i, \operatorname{such} \text { that } \lambda_{i}>0\right)=\operatorname{rank} \mathbf{X} .
$$

If we denote $V_{i}=\mathbf{X}^{\prime} U_{i} / \sqrt{\lambda}_{i}$, then the SVD of the trajectory matrix can be written as:

$$
\mathbf{X}=\mathbf{X}_{1}+\cdots+\mathbf{X}_{d}
$$

where $\mathbf{X}_{i}=\sqrt{\lambda_{i}} U_{i} V_{i}^{\prime}(i=1, \ldots, d)$. The matrices $\mathbf{X}_{i}$ are elementary matrices as they have rank $1, U_{i}$ and $V_{i}$ denotes the left and right eigenvectors of the trajectory matrix. The collection $\left(\sqrt{\lambda}_{i}, U_{i}, V_{i}\right)$ is called the $i$-th eigentriple of the matrix $\mathbf{X}, \sqrt{\lambda}_{i}(i=1, \ldots, d)$ are the singular values of the matrix $\mathbf{X}$ and the set $\left\{\sqrt{\lambda}_{i}\right\}$ is called the spectrum of the matrix $\mathbf{X}$. The expansion (1) is said to be uniquely defined if all the eigenvalues have a multiplicity of one.

\section{Stage 2: Reconstruction}

\section{1st step: Grouping}

At the first step in the second stage we split the elementary matrices $\mathbf{X}_{i}$ into several groups and sum the matrices within each group. This is referred to as the grouping step. Denote $I=\left\{i_{1}, \ldots, i_{p}\right\}$ as a group of indices $i_{1}, \ldots, i_{p}$. Then the matrix $\mathbf{X}_{I}$ corresponding to the group $I$ can be defined as $\mathbf{X}_{I}=\mathbf{X}_{i_{1}}+\cdots+\mathbf{X}_{i_{p}}$. The spilt of the set of indices $J=1, \ldots, d$ into the disjoint subsets $I_{1}, \ldots, I_{m}$ corresponds to the representation

$$
\mathbf{X}=\mathbf{X}_{I_{1}}+\cdots+\mathbf{X}_{I_{m}} .
$$

The procedure of choosing the sets $I_{1}, \ldots, I_{m}$ is called the eigentriple grouping. For a given group $I$ the contribution of the component $\mathbf{X}_{I}$ into the expansion (1) is measured by the share of the corresponding eigenvalues: $\sum_{i \in I} \lambda_{i} / \sum_{i=1}^{d} \lambda_{i}$. 


\section{2nd step: Diagonal Averaging}

Here we perform diagonal averaging in order to transform each matrix $I$ into a time series, which is an additive component of the initial series $Y_{T}$. For example, suppose $z_{i j}$ stands for an element of a matrix $\mathbf{Z}$, then the $k$-th term of the resulting series is obtained by averaging $z_{i j}$ over all $i, j$ such that $i+j=k+2$. This procedure is also known as Hankelization of the matrix $\mathbf{Z}$. The output of the Hankelization of a matrix $\mathbf{Z}$ is the Hankel matrix $\mathcal{H} \mathbf{Z}$, which is the trajectory matrix corresponding to the series obtained as a result of the diagonal averaging. In its turn, the Hankel matrix $\mathcal{H} \mathbf{Z}$ uniquely defines the series by relating the value in the diagonals to the values in the series. By applying the Hankelization procedure to all matrix components of (2), we obtain another expansion:

$$
\mathbf{X}=\widetilde{\mathbf{X}}_{I_{1}}+\ldots+\widetilde{\mathbf{X}}_{I_{m}}
$$

where $\widetilde{\mathbf{X}}_{I_{1}}=\mathcal{H} \mathbf{X}$. This is equivalent to the decomposition of the initial series $Y_{N}=\left(y_{1}, \ldots, y_{N}\right)$ into a sum of $m$ series:

$$
y_{n}=\sum_{k=1}^{m} \widetilde{y}_{n}^{(k)}
$$

where $\widetilde{Y}_{N}^{(k)}=\left(\widetilde{y}_{1}^{(k)}, \ldots, \widetilde{y}_{N}^{(k)}\right)$ corresponds to the matrix $\mathbf{X}_{I_{k}}$.

In the past, the selection of SSA choices of $L$ and $r$, such that they are optimal, has been a major issue. However, in this paper, we use the Root Mean Squared Error (RMSE) criterion (see, Section 4.2) to determine the optimal $L$ for decomposing the U.S. Tourist arrivals series, and the optimal $r$ for reconstructing the less noisy series which can then be used for forecasting (it is also possible to use any other criteria for minimising the forecasting error as explained below in the forecasting algorithm). Accordingly, we look for the combination of $L$ and $r$ which provides the lowest RMSE, and this in turn represents the optimal decomposition and reconstruction choices for the SSA model. ${ }^{1}$ Noteworthy is the fact that unlike with parameters of ARIMA, ETS and NN models, these optimal VSSA choices remain fixed for the respective horizon. The automated VSSA code is able to perform this task by evaluating all possible SSA choices for a given time series. The resulting optimal VSSA choices are presented in Table 3 for total U.S. Tourist arrivals and Table 8 for U.S. Tourist arrivals by country of origin. As such, this paper adopts basic VSSA with optimal choices for forecasting U.S. Tourist arrivals. Below, we provide a concise explanation of the VSSA forecasting algorithm that has been used for obtaining forecasts for U.S. Tourist arrivals.

1. Consider a real-valued nonzero time series (for example, Total U.S. Tourist arrivals) $Y_{N}=$ $\left(y_{1}, \ldots, y_{N}\right)$ of length $N$.

2. Divide the time series into two parts; $\frac{2}{3}^{\text {rd }}$ of observations for training and validating the VSSA model and $\frac{1}{3}^{\text {rd }}$ for testing the forecast accuracy. This is a common and widely accepted practice, visible in a majority of forecasting algorithms.

3. Use the training data to construct the trajectory matrix $\mathbf{X}=\left(x_{i j}\right)_{i, j=1}^{L, K}=\left[X_{1}, \ldots, X_{K}\right]$, where $X_{j}=\left(y_{j}, \ldots, y_{L+j-1}\right)^{T}$ and $K=N-L+1$. Initially, we begin with $L=2(2 \leq L \leq$ $\frac{N}{2}$ ) and in the process, evaluate all possible values of $L$ for $Y_{N}$.

4. Obtain the SVD of $\mathbf{X}$ by calculating $\mathbf{X} \mathbf{X}^{T}$ for which $\lambda_{1}, \ldots, \lambda_{L}$ denotes the eigenvalues in decreasing order $\left(\lambda_{1} \geq \ldots \lambda_{L} \geq 0\right)$ and by $U_{1}, \ldots, U_{L}$ the corresponding eigenvectors. The output of this stage is $\mathbf{X}=\mathbf{X}_{1}+\ldots+\mathbf{X}_{L}$ where $\mathbf{X}_{i}=\sqrt{\lambda_{i}} U_{i} V_{i}^{T}$ and $V_{i}=\mathbf{X}^{T} U_{i} / \sqrt{\lambda}_{i}$.

\footnotetext{
${ }^{1}$ The optimal SSA code used in this study is available upon request.
} 
5. Evaluate all possible combinations of $r(1 \leq r \leq L-1)$ singular values (step by step) for the selected $L$ and split the elementary matrices $\mathbf{X}_{i}(i=1, \ldots, L)$ into several groups and sum the matrices within each group.

6. Perform diagonal averaging to transform the matrix with the selected $r$ singular values into a Hankel matrix which can then be converted into a time series (the steps up to this stage filters the noisy series). The output is a filtered series that can be used for forecasting.

7. Set $v^{2}=\pi_{1}^{2}+\ldots+\pi_{r}^{2}$, where $\pi_{i}$ represents the final component of the eigenvector $U_{i}$ $(i=1, \ldots, r)$. Assume that, $e_{L}=(0,0 \ldots, 1)$ is not a component of the linear space $\mathfrak{L}_{r}$, which implies $\mathfrak{L}_{r}$ is not a vertical space.

8. Consider the matrix $\Pi=\mathbf{V}^{\nabla}\left(\mathbf{V}^{\nabla}\right)^{T}+\left(1-v^{2}\right) A A^{T}$, where $A=\left(a_{1}, \ldots, a_{L-1}\right)=\sum_{i=1}^{r} \pi_{i} U_{i}^{\nabla} /(1-$ $\left.v^{2}\right)$ and $\mathbf{V}^{\nabla}=\left[U_{1}^{\nabla}, \ldots, U_{r}^{\nabla}\right]$, where $\mathbf{V}^{\nabla}$ is the first $L-1$ components.

9. Next, consider the linear operator $\theta^{(v)}: \mathfrak{L}_{r} \mapsto \mathbf{R}^{L}$, where $\theta^{(v)} U=\left(\begin{array}{c}\Pi U^{\nabla} \\ A^{T} U^{\nabla}\end{array}\right)$.

10. Then, define vector $Z_{i}$ after grouping and eliminating noise components, such that

$$
Z_{i}= \begin{cases}\widetilde{X}_{i} & \text { for } i=1, \ldots, K \\ \theta^{(v)} Z_{i-1} & \text { for } i=K+1, \ldots, K+h+L-1,\end{cases}
$$

where, $\widetilde{X}_{i}$ 's are the reconstructed columns of the trajectory matrix.

11. Construct the matrix $\mathbf{Z}=\left[Z_{1}, \ldots, Z_{K+h+L-1}\right]$ and perform diagonal averaging to obtain a new series $y_{1}, \ldots, y_{N+h+L-1}$, where $y_{N+1}, \ldots, y_{N+h}$ forms the $h$ terms of the SSA Vector forecast.

12. Define a loss function $\mathcal{L}$.

13. When forecasting a series $Y_{N} h$-step ahead, the forecast error is minimised by setting $\mathcal{L}\left(X_{K+h}-\hat{X}_{K+h}\right)$ where the vector $\hat{X}_{K+h}$ contains the $h$-step ahead forecasts obtained using the VSSA forecasting algorithm.

14. Find the combination of $L$ and $r$ which minimises $\mathcal{L}$ and thus represents the optimal VSSA choices.

15. Finally use the optimal $L$ to decompose the series comprising of the training and validation set, and then select $r$ singular values for reconstructing the less noisy time series, and use this newly reconstructed series for forecasting the remaining $\frac{1}{3}^{\text {rd }}$ observations.

\section{The Data and Measures for Evaluating Forecast Accuracy}

\subsection{The Data}

This study uses monthly U.S. Tourist arrivals data from January 1996 to November 2012 obtained via the U.S. Department of Commerce: Office of Travel \& Tourism Industries ${ }^{2}$. Table 1 provides some descriptives of the data. According to the data, average total tourist arrivals into the U.S. between January 1996-November 2012 has been 3,798,000. The maximum number

\footnotetext{
${ }^{2}$ http://travel.trade.gov/research/monthly/arrivals/
} 
of tourist arrivals during the period of concern is recorded at 7,249,000 in July 2012, whilst the minimum number of arrivals was 2,096,000 in November 2001. On average, the lowest tourist arrivals into U.S. has been recorded from Africa whilst Canada accounts for an average of 1,346,000 tourist arrivals which is the highest influx of tourists into U.S. from a single country. The skewness statistic indicates that all time series analysed in this study are in fact skewed and not normally distributed. An analysis of the kurtosis suggests that all the series have Platykurtic distributions except for Italy which has a Leptokurtic distribution. Accordingly, this information tells us that the Italian time series for tourist arrivals into U.S. has a high probability for extreme values with thicker tails and values concentrated around the mean whilst all other time series for U.S. Tourist arrivals have a lesser probability for extreme values in comparison to a normal distribution and consist of values which have a wider spread around the mean. In order to confirm the information provided through the skewness and kurtosis statistics, the data was tested for normality using the Shapiro-Wilk test. Accordingly, it was found that Western Europe, Total Overseas, Asia and Central America were in fact normally distributed at a $p$-value of 0.05 , and that the skewness indicators are thus reliable. Note that in this paper we have evaluated the ARIMA model with and without log transformations and the results reported in the next section are the best outcomes. 
Table 1: Descriptive statistics: U.S. Tourist arrivals (Jan. 1996 - Nov. 2012).

\begin{tabular}{|c|c|c|c|c|c|c|c|}
\hline Series & Mean & Median & Min. & Max. & Std. Dev. & Skewness & Kurtosis \\
\hline Total Arrivals & 3798000 & 3590000 & 2096000 & 7249000 & 994944 & 0.86 & 0.63 \\
\hline \multicolumn{8}{|l|}{ Arrivals by country } \\
\hline Canada & 1346000 & 1261000 & 727300 & 2945000 & 417184 & 1.07 & 1.24 \\
\hline Mexico & 491100 & 381000 & 67960 & 1668000 & 338299 & 1.25 & 0.74 \\
\hline Total Overseas & 1961000 & 1922000 & 1119000 & 3089000 & 382831 & 0.34 & -0.06 \\
\hline Western Europe & 859700 & 848600 & 418800 & 1320000 & 187797 & 0.14 & -0.50 \\
\hline Eastern Europe & 39000 & 36170 & 17610 & 76360 & 11875 & 0.69 & -0.15 \\
\hline Asia & 550500 & 547200 & 246500 & 934300 & 106414 & 0.22 & 0.71 \\
\hline Middle East & 51600 & 48300 & 22930 & 120200 & 17996 & 1.09 & 1.21 \\
\hline Africa & 22870 & 22540 & 7869 & 48080 & 6863 & 0.63 & 0.51 \\
\hline Oceania & 65190 & 65190 & 28090 & 165600 & 23470 & 1.26 & 1.81 \\
\hline South America & 215200 & 212100 & 98580 & 420300 & 68877 & 0.62 & 0.06 \\
\hline Central America & 59510 & 58540 & 29730 & 91860 & 12097 & 0.29 & -0.14 \\
\hline Caribbean & 97440 & 90670 & 48330 & 191100 & 31712 & 1.05 & 0.51 \\
\hline France & 86290 & 78450 & 36920 & 201800 & 31954 & 1.20 & 1.39 \\
\hline Germany & 136800 & 132800 & 54920 & 235600 & 39695 & 0.24 & -0.80 \\
\hline Italy & 51460 & 45640 & 17170 & 157400 & 23127 & 1.88 & 4.65 \\
\hline Netherlands & 41180 & 38950 & 20340 & 90430 & 12554 & 1.26 & 2.23 \\
\hline Spain & 36260 & 30700 & 13110 & 104600 & 16651 & 1.40 & 2.15 \\
\hline Sweden & 25820 & 24780 & 11070 & 51560 & 7680 & 0.84 & 0.96 \\
\hline Switzerland & 29090 & 27630 & 13270 & 74220 & 10514 & 1.24 & 2.22 \\
\hline United Kingdom & 338400 & 349300 & 164300 & 475400 & 64735 & -0.46 & -0.34 \\
\hline Japan & 331200 & 317700 & 141600 & 549100 & 80225 & 0.36 & -0.21 \\
\hline South Korea & 62490 & 56080 & 19510 & 130300 & 22956 & 0.78 & 0.24 \\
\hline PRC \& Hongkong & 46480 & 38570 & 11480 & 207000 & 28966 & 2.63 & 8.62 \\
\hline ROC (Taiwan) & 27830 & 26480 & 9451 & 63400 & 10223 & 0.90 & 0.70 \\
\hline Australia & 51380 & 44940 & 21000 & 142400 & 20462 & 1.41 & 2.17 \\
\hline Argentina & 30780 & 31230 & 9279 & 64240 & 13845 & 0.25 & -1.00 \\
\hline Brazil & 65960 & 57190 & 18680 & 171000 & 34633 & 1.11 & 0.69 \\
\hline Colombia & 31810 & 29370 & 11110 & 74670 & 12050 & 0.79 & 0.18 \\
\hline Venezuela & 39330 & 36370 & 15780 & 86160 & 14841 & 0.93 & 0.51 \\
\hline
\end{tabular}

Next we test the U.S. Tourist arrivals series for unit root problems as certain external shocks such as recessions (for example) are infamous for making a time series nonstationary in mean and variance, thereby creating a structural break in the series. In Table 2 we report the findings from the Bai and Perron (2003) test for structural breaks in the U.S. Tourist arrivals series. Whilst analysing the causes and reasons behind these structural breaks are beyond the mandate of this paper, we will endeavour to outline certain observations. Firstly, we can see that based on the Bai and Perron (2003) test, the time series relating to tourist arrivals from the Caribbean is the only series that has not been affected by structural breaks. Secondly, except for Canada, Mexico, Africa, Central America, Germany, Italy, Spain, United Kingdom, South Korea, PRC \& Hongkong, Australia and Colombia, all other time series considered in this study are affected by a structural break in the year 2001. Interestingly, there are no structural breaks captured beyond 2010, and thus we conclude that either the impact of $9 / 11$ is masked in this level of data, or its impact was not major enough to cause a structural break in tourist arrivals into U.S. The National Bureau of Economic Research ${ }^{3}$. reports the U.S. experienced its last recession

\footnotetext{
${ }^{3}$ http://www.nber.org/cycles/cyclesmain.html
} 
beginning December 2007. The Bai and Perron (2003) test shows there has been a lagged impact of this recession on U.S. Tourist arrivals with all series reporting structural breaks in 2010 with the exception of Mexico, Western Europe, Africa, Germany, Italy, Netherlands, Spain, United Kingdom, Japan, ROC (Taiwan), Colombia and Venezuela. Finally, in terms of U.S. Tourist arrivals by country of origin, the most number of structural breaks visible in a time series is seen in tourist arrivals from Brazil. The presence of structural breaks would also enable us to ascertain the sensitiveness of the various forecasting techniques to such break points.

Table 2: Break points in U.S. Tourist arrivals time series.

\begin{tabular}{ll}
\hline Series & Structural Break \\
\hline Total Arrivals & $2001(8), 2004(3), 2007(2), 2010(2)$ \\
Arrivals by country & \\
Canada & $2006(2), 2010(2)$ \\
Mexico & $1998(6), 2006(3), 2009(12)$ \\
Total Overseas & $2001(8), 2004(3), 2007(4), 2010(4)$ \\
Western Europe & $2001(8), 2004(2), 2007(6)$ \\
Eastern Europe & $2001(8), 2004(5), 2007(4), 2010(5)$ \\
Asia & $2001(8), 2004(4), 2010(4)$ \\
Middle East & $1999(2), 2001(8), 2006(5), 2010(5)$ \\
Africa & $2008(4)$ \\
Oceania & $2001(9), 2004(3), 2007(3), 2010(4)$ \\
South America & $2001(8), 2007(5), 2010(5)$ \\
Central America & $1998(6), 2001(8), 2007(4)$ \\
Caribbean & No structural break in series. \\
France & $2001(8), 2007(3), 2010(3)$ \\
Germany & $2000(10), 2007(2)$ \\
Italy & $2007(6)$ \\
Netherlands & $2001(8), 2007(3)$ \\
Spain & $2007(5)$ \\
Sweden & $2001(6), 2004(2), 2007(2), 2010(5)$ \\
Switzerland & $2001(7), 2007(3), 2010(5)$ \\
United Kingdom & $1998(6), 2008(12)$ \\
Japan & $2001(8)$ \\
South Korea & $2005(4), 2010(4)$ \\
PRC \& Hongkong & $2007(4), 2010(5)$ \\
ROC (Taiwan) & $2001(8)$ \\
Australia & $2005(4), 2010(4)$ \\
Argentina & $2001(8), 2006(12), 2010(4)$ \\
Brazil & $1999(1), 2001(7), 2005(4), 2007(11), 2010(5)$ \\
Colombia & $2009(5)$ \\
Venezuela & $2001(12), 2007(6)$ \\
&
\end{tabular}

In Table 3 we present the model parameters (SSA choices) for each of the forecasting techniques considered in this study for forecasting total U.S. Tourist arrivals at horizons of $h=1$, 3, 6 and 12 months ahead. It is important to note that each of the techniques have chosen the 
model parameters (SSA choices) automatically using the respective algorithms (see, Section 3) to provide the best possible modelling and forecast for U.S. Tourist arrivals.

Table 3: Forecasting model parameters for total U.S. Tourist arrivals.

\begin{tabular}{ccccc}
\hline$h$ & ARIMA & $\operatorname{ETS}(\alpha, \sigma)$ & $\operatorname{NN}(p, P, k)$ & $\operatorname{SSA}(L, r)$ \\
\hline 1 & order $(2,0,1)$ seasonal $(1,1,2)$ & $(0.87,0.1245)^{M}$ & $\operatorname{NNAR}(2,1,1)$ & $(38,17)$ \\
3 & order $(2,0,1)$ seasonal $(1,1,2)$ & $(0.86,0.1241)^{M}$ & $\operatorname{NNAR}(2,1,1)$ & $(25,14)$ \\
6 & order(2,0,1)seasonal $(1,1,2)$ & $(0.87,0.1239)^{M}$ & $\operatorname{NNAR}(2,1,1)$ & $(29,21)$ \\
12 & order $(2,0,1)$ seasonal $(1,1,2)$ & $(0.86,0.1231)^{M}$ & $\operatorname{NNAR}(2,1,1)$ & $(15,6)$ \\
24 & order $(2,0,1)$ seasonal $(1,1,2)$ & $(0.85,0.1586)^{M}$ & $\operatorname{NNAR}(2,1,1)$ & $(40,25)$ \\
36 & order $(2,0,1)$ seasonal $(1,1,2)$ & $(0.92,0.1531)^{M}$ & $\operatorname{NNAR}(2,1,1)$ & $(48,6)$ \\
\hline \multicolumn{5}{c}{ Note: $^{M}$ is an ETS model with multiplicative seasonality. }
\end{tabular}

$p$ is the number of lagged inputs, $P$ is the automatically selected value for seasonal time series, and $k$ is the number of nodes in the hidden layer. $L$ is the window length and $r$ is the number of eigenvalues.

Next, we consider the VSSA decompositions which is an integral part of the SSA process. The weighted correlation ( $w$-correlation) statistic is used to show the appropriateness of the various decompositions achieved by SSA (see, Table 3 and Table 7). As mentioned in Golyandina, Nekrutkin, and Zhigljavsky (2001), the $w$-correlation statistic which shows the dependence between two time series can be calculated as:

$$
\rho_{12}^{(w)}=\frac{\left(Y_{N}^{(1)}, Y_{N}^{(2)}\right)_{w}}{\left\|Y_{N}^{(1)}\right\|_{w}\left\|Y_{N}^{(2)}\right\|_{w}},
$$

where $Y_{N}^{(1)}$ and $Y_{N}^{(2)}$ are two time series, $\left\|Y_{N}^{(i)}\right\|_{w}=\sqrt{\left(Y_{N}^{(i)}, Y_{N}^{(i)}\right)_{w}},\left(Y_{N}^{(i)}, Y_{N}^{(j)}\right)_{w}=\sum_{k=1}^{N} w_{k} y_{k}^{(i)} y_{k}^{(j)}$ $(i, j=1,2), w_{k}=\min \{k, L, N-k\} \quad$ (here, assume $\left.L \leq N / 2\right)$.

Accordingly, if the $w$-correlation between two reconstructed components are close to 0 , this implies that the corresponding series are $w$-orthogonal and in turn we know the two components are well separable (Hassani, Heravi, \& Zhigljavsky, 2009). In Table 3 we calculate the $w$ correlations for all the decompositions by comparing the two components of signal and noise. Here, we use as signal the reconstructed series containing optimal $r$ components and select the remaining $r$ (which does not belong to the reconstruction) as noise. The results indicate that all $w$-correlations are close to 0 which in turn suggests that we have achieved a sound decomposition using the VSSA forecasting algorithm (see, Section 3.4). In other words, these $w$-correlations indicate that the VSSA forecasting algorithm works exceedingly well at separating the noise from the signal. 
Table 4: $W$-correlations between signal and residuals for U.S. Tourist arrivals.

\begin{tabular}{|c|c|c|c|c|c|c|}
\hline Series & 1 & 3 & 6 & 12 & 24 & 36 \\
\hline Total U.S. Tourist Arrivals & 0.007 & 0.009 & 0.009 & 0.012 & 0.008 & 0.009 \\
\hline \multicolumn{7}{|c|}{ U.S. Tourist Arrivals by country } \\
\hline Canada & 0.013 & 0.010 & 0.010 & 0.028 & 0.010 & 0.012 \\
\hline Mexico & 0.020 & 0.020 & 0.021 & 0.047 & 0.032 & 0.035 \\
\hline Total Overseas & 0.009 & 0.009 & 0.014 & 0.014 & 0.008 & 0.006 \\
\hline Western Europe & 0.010 & 0.014 & 0.015 & 0.019 & 0.024 & 0.012 \\
\hline Eastern Europe & 0.020 & 0.016 & 0.014 & 0.015 & 0.022 & 0.020 \\
\hline Asia & 0.008 & 0.008 & 0.008 & 0.017 & 0.007 & 0.006 \\
\hline Middle East & 0.027 & 0.047 & 0.044 & 0.029 & 0.022 & 0.024 \\
\hline Africa & 0.019 & 0.020 & 0.015 & 0.031 & 0.013 & 0.010 \\
\hline Oceania & 0.010 & 0.009 & 0.014 & 0.018 & 0.007 & 0.007 \\
\hline South America & 0.012 & 0.019 & 0.023 & 0.016 & 0.020 & 0.023 \\
\hline Central America & 0.013 & 0.016 & 0.014 & 0.021 & 0.012 & 0.016 \\
\hline Caribbean & 0.021 & 0.021 & 0.031 & 0.051 & 0.034 & 0.019 \\
\hline France & 0.014 & 0.027 & 0.040 & 0.015 & 0.014 & 0.015 \\
\hline Germany & 0.015 & 0.015 & 0.014 & 0.017 & 0.017 & 0.017 \\
\hline Italy & 0.026 & 0.026 & 0.026 & 0.016 & 0.035 & 0.024 \\
\hline Netherlands & 0.016 & 0.018 & 0.018 & 0.018 & 0.027 & 0.014 \\
\hline Spain & 0.030 & 0.014 & 0.031 & 0.027 & 0.016 & 0.027 \\
\hline Sweden & 0.012 & 0.012 & 0.012 & 0.011 & 0.012 & 0.017 \\
\hline Switzerland & 0.024 & 0.016 & 0.021 & 0.017 & 0.020 & 0.020 \\
\hline United Kingdom & 0.013 & 0.016 & 0.015 & 0.013 & 0.012 & 0.016 \\
\hline Japan & 0.009 & 0.015 & 0.008 & 0.009 & 0.07 & 0.012 \\
\hline South Korea & 0.016 & 0.016 & 0.012 & 0.016 & 0.016 & 0.012 \\
\hline PRC \& Hongkong & 0.025 & 0.051 & 0.022 & 0.030 & 0.025 & 0.022 \\
\hline ROC (Taiwan) & 0.019 & 0.031 & 0.025 & 0.025 & 0.015 & 0.015 \\
\hline Australia & 0.011 & 0.011 & 0.011 & 0.011 & 0.011 & 0.011 \\
\hline Argentina & 0.028 & 0.010 & 0.046 & 0.029 & 0.007 & 0.010 \\
\hline Brazil & 0.025 & 0.023 & 0.026 & 0.027 & 0.030 & 0.027 \\
\hline Colombia & 0.022 & 0.023 & 0.012 & 0.012 & 0.019 & 0.038 \\
\hline Venezuela & 0.026 & 0.025 & 0.026 & 0.046 & 0.022 & 0.021 \\
\hline
\end{tabular}

The U.S. Tourist arrivals series exhibits several seasonal patterns. In order to illustrate SSA's capabilities at extracting various seasonal patterns in U.S. Tourist arrivals we present in Figure 2, as an example, the in-sample decomposition of total U.S. Tourist arrivals at $h=1$ step ahead. Firstly we can observe the extracted trend in U.S. Tourist arrivals which corresponds with the total arrivals pattern and clearly shows the general trend of increase and decreasing tourist arrivals over time. Also interesting is the difference between the four month and twelve month seasonal components. The 4 month seasonal component is increasing over time whilst the 12 month seasonal component is seen to be decreasing over time. Furthermore, there is more fluctuation in the 4 month seasonal component of total U.S. Tourist arrivals in comparison to the 12 month component. 

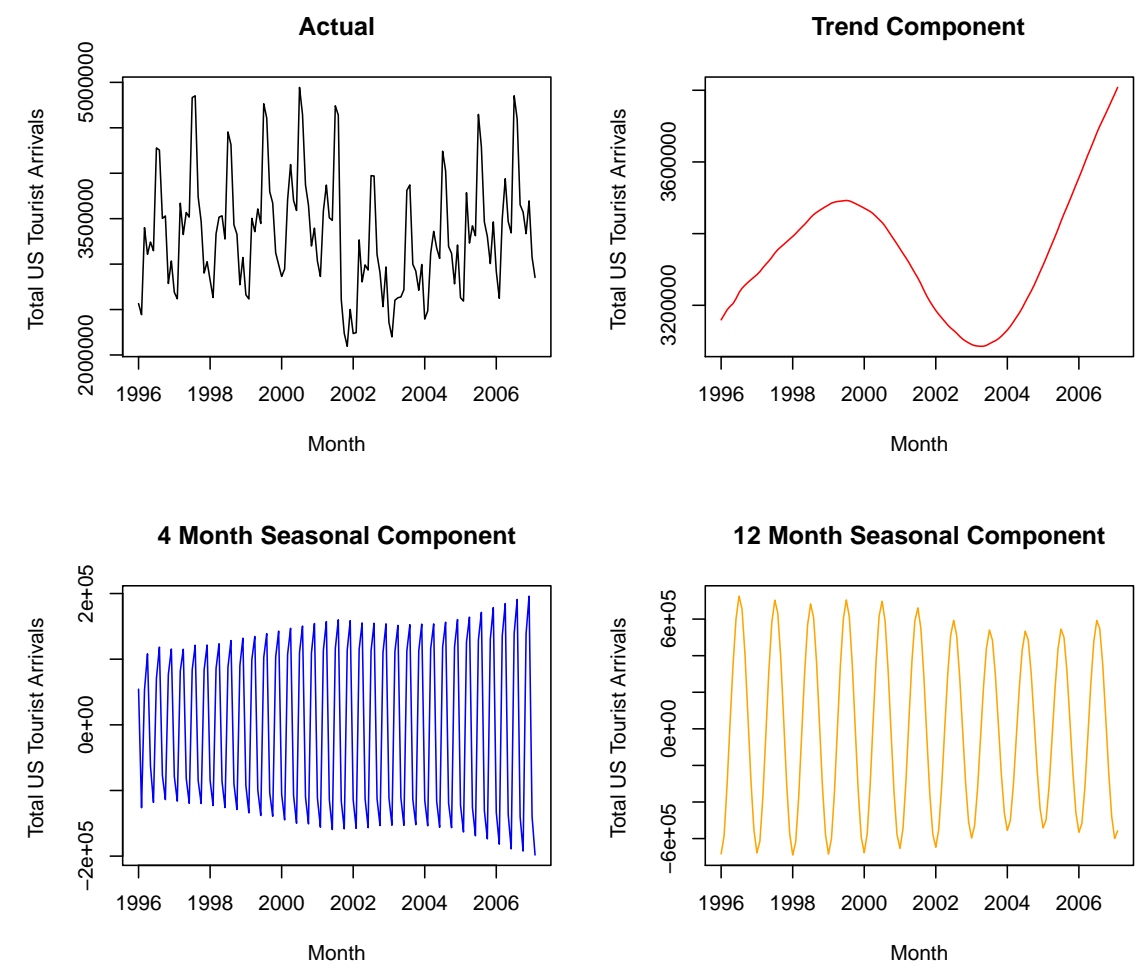

Figure 2: In-sample SSA decomposition of total monthly U.S. Tourist arrivals at $h=1$ step ahead.

\subsection{Measures for Evaluating the Forecast Accuracy \\ Root Mean Squared Error (RMSE)}

The RMSE is now a popular measure for forecast accuracy, and is one of the most frequently cited measures in forecasting literature (see, for example, Zhang et al. 1998; Hassani, Heravi, \& Zhigljavsky, 2009; Hassani, Heravi, \& Zhigljavsky, 2012; Hassani \& Mahmoudvand, 2013; Beneki \& Silva, 2013). Here, in order to save space, we only provide the RMSE ratios of SSA to that of ETS:

$$
\operatorname{RRMSE}=\frac{S S A}{E T S}=\frac{\left(\sum_{i=1}^{N}\left(\widehat{y}_{T+h, i}-y_{T+h, i}\right)^{2}\right)^{1 / 2}}{\left(\sum_{i=1}^{N}\left(\widetilde{y}_{T+h, i}-y_{T+h, i}\right)^{2}\right)^{1 / 2}},
$$

where, $\widehat{y}_{T+h}$ is the $h$-step ahead forecast obtained by SSA, $\widetilde{y}_{T+h}$ is the $h$-step ahead forecast from the ETS model, and $N$ is the number of the forecasts. If $\frac{S S A}{E T S}$ is less than 1, then the SSA outperforms ETS by $1-\frac{S S A}{E T S}$ percent.

\section{Mean Absolute Percentage Error (MAPE)}

The MAPE measure is also quoted in this paper as it is a widely understood criterion for evaluating forecast accuracy. In brief, the lower the MAPE result, the better the forecast. 


$$
\mathrm{MAPE}=\frac{1}{N} \sum_{t=1}^{N}\left|100 \times \frac{y_{T+h}-\widehat{y}_{T+h, i}}{y_{T+h}}\right|,
$$

where $y_{T+h}$ represents the actual data corresponding to the $h$ step ahead forecast, and $\widehat{y}_{T+h, i}$ is the $h$ step ahead forecasts obtained from a particular forecasting model.

\section{Direction of Change (DC)}

The DC criterion is a measure of the percentage of forecasts that accurately predict the direction of change (Hassani, Heravi, \& Zhigljavsky, 2012). DC is an equally important measure, as the RMSE, for evaluating the forecasting performance of tourism demand models, because it is important that for example, when the actual series is illustrating an upwards trend, the forecast is able to predict that upward trend and vice versa. Here, the concept of DC is explained in brief, and in doing so we mainly follow Hassani, Heravi, and Zhigljavsky (2012). In the univariate case, for forecasts obtained using $X_{T}$, let $D_{X i}$ be equal to 1 if the forecast is able to correctly predict the actual direction of change and 0 otherwise. Then, $\tilde{D}_{X}=\sum_{i=1}^{n} D_{X i} / n$ shows the proportion of forecasts that correctly identify the direction of change in the actual series.

\section{$5 \quad$ Empirical Results}

We select $\frac{2}{3}^{\text {rd }}$ of the data as in-sample for model training and validation, and set aside $\frac{1}{3}^{\text {rd }}$ of the data as out-of-sample for evaluating the forecasting accuracy. The data was forecasted at horizons of $h=1,3,6,12,24$ and 36 steps ahead which corresponds to $1,3,6,12,24$ and 36 month ahead forecasts. These forecasting horizons have been considered because for the tourism industry, horizons beyond 12 months are considered to be long term. Moreover, both short and long run forecasts are vital for this sector as a country needs to be geared to accommodate the tourists and planning of large scale building works or the purchase of new aircrafts for example would require managerial decisions to be made well in advance. Therefore, in this paper we are effectively evaluating the performance of the forecasting models both in the short and long run in terms of obtaining forecasts for U.S. tourist arrivals. We first analyse total U.S. Tourist arrivals. Table 5 reports the RMSE and MAPE results for the out-of-sample forecasts of total U.S. Tourist arrivals using SSA, ARIMA, ETS and NN. In order to ensure the parametric models are correctly specified we carried out a Ljung-Box test on the residuals for autocorrelation and the results indicated that the residuals are independently distributed at a $p$-value of 0.05 , and are there for not autocorrelated.

Table 5: Out-of-sample RMSE(MAPE) results for total U.S. Tourist arrivals.

\begin{tabular}{cccccccc}
\hline$h$ & ARIMA & ETS & NN & SSA & $\frac{S S A}{A R I M A}$ & $\frac{S S A}{E T S}$ & $\frac{S S A}{N N}$ \\
\hline 1 & $601512(9 \%)$ & $760599(13 \%)$ & $1147080(19 \%)$ & $242601(4 \%)$ & $0.40^{*}$ & $0.32^{*}$ & $0.21^{*}$ \\
3 & $720751(11 \%)$ & $723556(13 \%)$ & $1124242(19 \%)$ & $316049(6 \%)$ & $0.44^{*}$ & $0.44^{*}$ & $0.28^{*}$ \\
6 & $738630(12 \%)$ & $1037666(20 \%)$ & $1180780(19 \%)$ & $445614(8 \%)$ & $0.60^{*}$ & $0.43^{*}$ & $0.38^{*}$ \\
12 & $937129(14 \%)$ & $1097366(17 \%)$ & $1385339(23 \%)$ & $517912(9 \%)$ & $0.55^{*}$ & $0.47^{*}$ & $0.37^{*}$ \\
24 & $1136616(19 \%)$ & $1300442(20 \%)$ & $1780513(30 \%)$ & $526323(9 \%)$ & $0.46^{*}$ & $0.40^{*}$ & $0.30^{*}$ \\
36 & $1002685(17 \%)$ & $1149585(18 \%)$ & $1684799(24 \%)$ & $605448(9 \%)$ & $0.60^{*}$ & $0.53^{*}$ & $0.36^{*}$ \\
\hline Average & $856221(14 \%)$ & $1011536(17 \%)$ & $1383792(22 \%)$ & $442325(8 \%)$ & 0.52 & 0.44 & 0.32 \\
\hline \multicolumn{7}{c}{ Note:* indicates results are statistically significant based on Diebold-Mariano at $p=0.05}$.
\end{tabular}


Firstly, based on the MAPE criterion reported in Table 5, we can see that the Neural Network model is the worst performer at all horizons with an overall average MAPE of $22 \%$ at forecasting total U.S. Tourist arrivals. Interestingly the SSA technique is the only model which reports MAPE values below $10 \%$ at all horizons and is in turn the model providing the most accurate forecasts for total U.S. Tourist arrivals with a comparatively low average MAPE of 8\%. Based on the MAPE we are also able to identify that the ARIMA model is the second best model for forecasting total U.S. Tourist arrivals as its average MAPE of $14 \%$ is lower than the ETS model's average MAPE of $17 \%$. Moreover, it is interesting to note that the SSA model's MAPE remains approximately constant over the forecasting horizons of $h=12,24$ and 36 months ahead, and thereby portrays SSA's capabilities of providing comparatively stable and more accurate forecasts in the long run. The remainder of the analysis focusses on the RMSE criterion for evaluating forecast accuracy.

It is evident from Table 5 that based on the RMSE criterion, SSA outperforms ARIMA, ETS and Neural Networks comfortably by recording the lowest forecasting error for total U.S. Tourist arrivals at all horizons. The RRMSE statistic shows that SSA is $60 \%, 56 \%, 40 \%, 45 \%, 54 \%$ and $40 \%$ better than ARIMA at forecasting total U.S. Tourist arrivals at $h=1,3,6,12,24$ and 36 months ahead respectively. Likewise, in comparison to ETS, SSA is $68 \%, 56 \%, 57 \%, 53 \%, 60 \%$ and $47 \%$ better at $h=1,3,6,12,24$ and 36 steps ahead respectively. Finally we analyse the forecasting results between SSA and the Neural Network model. Accordingly we can conclude that the SSA model is $79 \%, 72 \%, 62 \%, 63 \%, 70 \%$ and $64 \%$ better than the feed-forward Neural Network model at $h=1,3,6,12,24$ and 36 months ahead respectively.

In order to ensure the results reported are not chance occurrences, we test the results further for statistical significance using the modified Diebold-Mariano test found in Harvey, Leybourne, and Newbold (1997). We find that all the RRMSE results are statistically significant at all horizons and thus provides concrete evidence for the inferences we have made. Finally, from Table 5 we can infer that when forecasting total U.S. Tourist arrivals, on average, the SSA model is $48 \%$ better than ARIMA, $56 \%$ better than ETS and $68 \%$ better than Neural Networks based on the forecasting accuracy. Note that initially, we normalized the data (where necessary) and evaluated the forecasting accuracy of the ARIMA model. ARIMA's forecasting results for U.S. Tourist arrivals were adversely affected following the data transformations. As such we have reported the results sans transformation which gives the best possible outcome for the ARIMA forecasts. The results from Table 5 also show that on average, ARIMA provides a better forecasting accuracy in comparison to ETS and Neural Networks for U.S. Tourist arrivals forecasting both in the short and long run, and is therefore chosen to be the second best model in general for this purpose. The feed-forward Neural Network model with one hidden layer provides the least favourable forecasts for total U.S. Tourist arrivals.

Thereafter, we use the Direction of Change (DC) criterion to evaluate the extent to which the forecasts from all models are able to predict the actual direction of change in total U.S. Tourist arrivals. Table 6 presents the DC results. Firstly, we see that only three outcomes are in fact statistically significant for DC. However, based on the criterion itself we could infer that SSA provides a more accurate prediction of direction of change in comparison to ARIMA at all horizons when forecasting total U.S. Tourist arrivals, and on average, SSA is able to provide a $83 \%$ accurate direction of change prediction whilst ARIMA can only provide a $63 \%$ accurate prediction of the direction of change in total U.S. Tourist arrivals. Likewise, in comparison to both ETS and Neural Networks, SSA provides a better prediction of the direction of change at all horizons. However ETS outperforms the ARIMA model in terms of DC at $h=3$ and 24 months ahead and the DC predictions of the NN model is better than ETS at $h=12$ and 24 steps ahead. Furthermore, at 36 steps ahead the SSA model obtains 100\% accurate DC 
predictions whilst ARIMA is able to report a significant $91 \%$ accuracy. Thus, it is clear that the SSA model stands out as the most superior model for forecasting total U.S. Tourist arrivals at all horizons based on the RMSE, RRMSE and DC criterions in comparison to the optimal ARIMA, ETS and Neural Network models. Furthermore, it is clear that the SSA model can pick up both short and long run fluctuations in total U.S. Tourist arrivals comparatively better than ARIMA, ETS and Neural Networks.

Table 6: Direction of change results for total U.S. tourist arrivals forecasts.

\begin{tabular}{ccccc}
\hline$h$ & ARIMA & ETS & NN & SSA \\
\hline 1 & $0.74^{*}$ & $0.57^{*}$ & 0.48 & 0.87 \\
3 & 0.70 & 0.73 & 0.57 & $0.85^{* *}$ \\
6 & 0.67 & 0.63 & 0.56 & 0.81 \\
12 & 0.47 & 0.36 & 0.45 & 0.66 \\
24 & 0.30 & 0.52 & 0.63 & $0.78^{*}$ \\
36 & $0.91^{* *}$ & 0.56 & 0.56 & $1.00^{*}$ \\
\hline Average & 0.63 & 0.56 & 0.54 & 0.83
\end{tabular}

Note:* indicates results are statistically significant based on a t-test at $p=0.05$.

$* *$ indicates results are statistically significant based on a t-test at $p=0.10$.

As an example of the out-of-sample forecasting capabilities of the selected models, and also to show the accuracy of the DC results, in Figure 2 we provide a graphical representation of the forecasting results at $h=24$ steps ahead for total U.S. Tourist arrivals. It is further evident from Figure 2 that both ETS and NN models experience great difficulty in picking up the seasonal fluctuations seen in the U.S. Tourist arrivals time series and that the NN model is indeed the worst performer in this case. The results from both Tables 5 and 6 proves that as the horizon increases from 1 month ahead to 24 months ahead, the forecasting performance of the parametric model (ARIMA), ETS and NN worsens immensely in comparison to that of the nonparametric model of SSA.

The initial results guided our interest towards evaluating the use of SSA for forecasting U.S. Tourist arrivals by country of origin. The total U.S. Tourist arrivals forecasting results show ARIMA to be the second best forecasting model in comparison to SSA, ETS and Neural Networks. As such, here we employ ARIMA as our benchmark as it is evident that ETS and feed-forward Neural Networks cannot provide accurate forecasts in this case. In Tables 7 and 8 we present the ARIMA parameters and Vector SSA choices which were used for forecasting U.S. Tourist arrivals by country of origin. It should be noted that we have once again reviewed the models are correctly specified via a Ljung-Box test for the independent distribution of residuals. Where the residuals were not found to be independently distributed (for example, in some cases at $h=24$ and 36 steps ahead this issue was experienced) we redefined the model parameters to ensure the model specification was valid. In most cases the test results indicated that the residuals were white noise at a $p$-value of 0.05 , and that no further model review was required. 


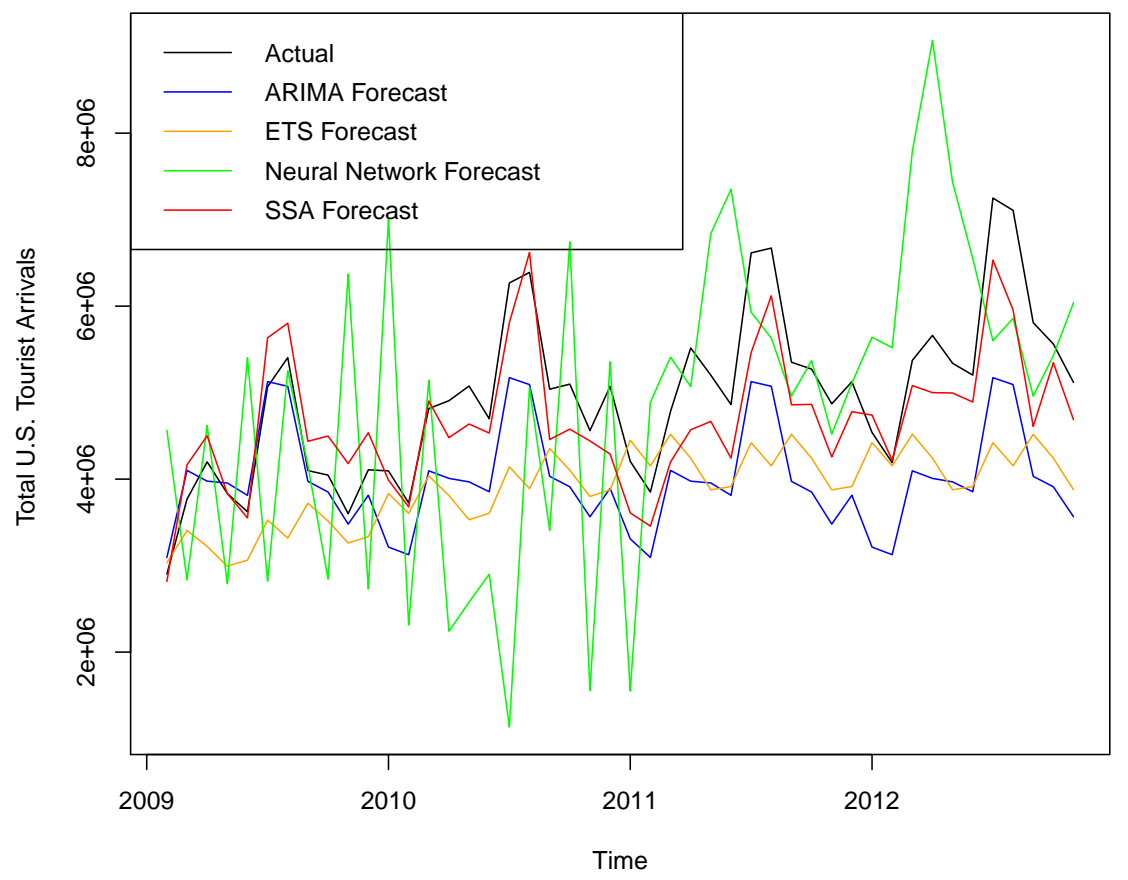

Figure 3: 24 months ahead forecast for U.S. Tourist arrivals (Feb. 2009 - Nov. 2012). 
Table 7: U.S. Tourist arrivals by country of origin - ARIMA model parameters.

\begin{tabular}{|c|c|c|c|c|c|c|}
\hline Series & 1 & 3 & 6 & 12 & 24 & 36 \\
\hline Canada & $(0,0,1) S$ & $(3,0,3) S$ & $(0,0,2) S$ & $(0,0,2) S$ & $(0,0,2) S$ & $(0,0,2) S$ \\
\hline Mexico & $(1,1,3) S$ & $(2,1,3) S$ & $(2,1,3) S$ & $(0,1,3) S$ & $(2,1,3) S$ & $(1,1,3) S$ \\
\hline Total Overseas & $(0,1,1) S 1$ & $(0,1,1) S 1$ & $(0,1,1) S 1$ & $(0,1,1) S 1$ & $(0,1,1) S 1$ & $(0,1,1) S 1$ \\
\hline Western Europe & $(1,0,0) S 2$ & $(1,0,0) S 2$ & $(1,0,0) S 2$ & $(1,0,0) S 2$ & $1,0,0) S 2$ & $1,0,0) S 2$ \\
\hline Eastern Europe & $(2,0,1) S 3$ & $(2,0,1) S 3$ & $(2,1,2) S 3$ & $(3,1,2) S 3$ & $(3,1,2) S 3$ & $(3,1,2) S 3$ \\
\hline Asia & $(0,1,0) S 2$ & $(0,1,0) S 2$ & $(0,1,0) S 2$ & $(0,1,0) S 2$ & $(0,1,0) S 2$ & $(0,1,0) S 2$ \\
\hline Middle East & $(2,0,1) S 1$ & $(2,0,1) S 1$ & $(2,0,1) S 1$ & $(2,0,1) S 1$ & $2,0,1) S 1$ & $2,0,1) S 1$ \\
\hline Africa & $(2,0,3) S 4$ & $(2,0,3) S 4$ & $(2,0,3) S 4$ & $(2,0,3) S 4$ & $(2,0,3) S 4$ & $(2,0,3) S 4$ \\
\hline Oceania & $(3,0,3) S 5$ & $(3,0,3) S 5$ & $(3,0,3) S 5$ & $(5,1,3) S 5$ & $(5,1,3) S 5$ & $(2,1,5) S 5$ \\
\hline South America & $(0,1,2) S 2$ & $(0,1,2) S 2$ & $(0,1,2) S 2$ & $(1,1,2) S 2$ & $(1,1,2) S 2$ & $(1,1,2) S 2$ \\
\hline Central America & $(2,1,1) S 6$ & $(2,1,1) S 6$ & $(2,1,1) S 6$ & $(2,1,1) S 6$ & $(2,1,1) S 6$ & $(2,1,1) S 6$ \\
\hline Caribbean & $(0,0,1) S 3$ & $(0,0,1) S 3$ & $(0,0,1) S 3$ & $(0,0,1) S 3$ & $(0,0,1) S 3$ & $(0,0,1) S 3$ \\
\hline France & $(1,1,1) S 1^{\dagger}$ & $(1,1,1) S 1^{\dagger}$ & $(1,1,1) S 1^{\dagger}$ & $(1,1,1) S 1^{\dagger}$ & $(2,0,0) S 1^{\dagger}$ & $(2,0,0) S 1^{\dagger}$ \\
\hline Germany & $(2,1,3) S 1$ & $(2,1,4) S 1$ & $(2,1,4) S 1$ & $(2,1,4) S 1$ & $(4,1,5) S 1$ & $(2,1,4) S 1$ \\
\hline Italy & $(2,0,2) S 3^{\dagger}$ & $(2,0,2) S 3^{\dagger}$ & $(2,0,2) S 3^{\dagger}$ & $(2,0,1) S 3^{\dagger}$ & $(2,0,5) S 3^{\dagger}$ & $(2,1,1) S 3^{\dagger}$ \\
\hline Netherlands & $(4,0,4) S 4$ & $(4,0,4) S 4$ & $(4,0,5) S 4$ & $(4,0,4) S 4$ & $(4,0,4) S 4$ & $(4,0,4) S 4$ \\
\hline Spain & $(3,0,3) S 5$ & $(3,0,3) S 5$ & $(2,1,3) S 5$ & $(2,1,1) S 5$ & $(1,1,1) S 5$ & $(1,1,1) S 5$ \\
\hline Sweden & $(2,1,1) S 3$ & $(2,1,1) S 3$ & $(2,1,1) S 3$ & $(2,1,1) S 3$ & $(2,1,3) S 3$ & $(2,1,4) S 3$ \\
\hline Switzerland & $(5,1,4) S 1$ & $(5,1,4) S 1$ & $(3,1,3) S 1$ & $(5,1,4) S 1$ & $(5,1,4) S 1$ & $(4,1,2) S 1$ \\
\hline UK & $(2,1,4) S 2$ & $(0,1,3) S 2$ & $(1,1,1 S 2)$ & $(2,1,5) S 2$ & $(2,1,5) S 2$ & $(2,1,4) S 2$ \\
\hline Japan & $(2,1,2) S 2$ & $(3,1,4) S 2$ & $(2,1,2) S 2$ & $(2,1,2) S 2$ & $(2,1,2) S 2$ & $(2,1,5) S 2$ \\
\hline South Korea & $(1,0,1) S 7^{\dagger}$ & $(1,0,1) S 7^{\dagger}$ & $(0,1,0) S 7^{\dagger}$ & $(0,1,0) S 7^{\dagger}$ & $(0,1,0) S 7^{\dagger}$ & $(0,1,0) S 7^{\dagger}$ \\
\hline PRC \& Hongkong & $(1,0,0) S 1^{*}$ & $(1,0,0) S 1^{*}$ & $(1,0,0) S 1^{*}$ & $(1,0,0) S 1$ & $(1,1,1) S 1$ & $(1,1,1) S 1$ \\
\hline $\mathrm{ROC}$ & $(4,1,2) S 1$ & $(4,1,2) S 1$ & $(4,1,2) S 1$ & $(5,1,2) S 1$ & $(5,1,3) S 1$ & $(4,1,3) S 1$ \\
\hline Australia & $(4,1,5) S 8$ & $(3,1,4) S 8$ & $(4,1,5) S 8$ & $(4,1,5) S 8$ & $(3,1,4) S 8$ & $(3,1,2) S 8$ \\
\hline Argentina & $(1,1,1) S 2$ & $(1,1,1) S 2$ & $(1,1,1) S 2$ & $(1,1,1) S 2$ & $(1,1,1) S 2$ & $(1,1,1) S 2$ \\
\hline Brazil & $(1,1,2) S 2$ & $(2,1,5) S 2$ & $(2,1,5) S 2$ & $(4,1,5) S 2$ & $(4,1,5) S 2$ & $(2,1,3) S 2$ \\
\hline Colombia & $(2,0,4) S 7$ & $(2,0,5) S 7$ & $(4,0,4) S 7$ & $(4,0,3) S 7$ & $(3,0,3) S 7$ & $(2,1,3) S 7$ \\
\hline Venezuela & $(3,1,1) S 5$ & $(3,1,1) S 5$ & $(3,1,5) S 5$ & $(3,1,1) S 5$ & $(3,1,1) S 5$ & $(3,1,1) S 5$ \\
\hline
\end{tabular}


Table 8: U.S. Tourist arrivals by country of origin - Vector SSA choices $(L, r)$.

\begin{tabular}{lcccccc}
\hline Series & 1 & 3 & 6 & 12 & 24 & 36 \\
\hline Canada & $(22,16)$ & $(28,19)$ & $(28,19)$ & $(16,9)$ & $(33,20)$ & $(36,15)$ \\
Mexico & $(5,1)$ & $(5,1)$ & $(4,1)$ & $(51,3)$ & $(39,9)$ & $(49,26)$ \\
Total Overseas & $(28,16)$ & $(29,16)$ & $(20,10)$ & $(18,11)$ & $(38,25)$ & $(50,29)$ \\
Western Europe & $(29,17)$ & $(23,14)$ & $(23,12)$ & $(19,14)$ & $(21,13)$ & $(28,21)$ \\
Eastern Europe & $(14,13)$ & $(22,11)$ & $(23,11)$ & $(20,14)$ & $(18,13)$ & $(49,5)$ \\
Asia & $(29,23)$ & $(25,22)$ & $(29,23)$ & $(23,11)$ & $(31,28)$ & $(49,40)$ \\
Middle East & $(24,15)$ & $(15,13)$ & $(17,13)$ & $(22,18)$ & $(44,36)$ & $(38,15)$ \\
Africa & $(18,14)$ & $(17,14)$ & $(24,20)$ & $(14,12)$ & $(47,17)$ & $(24,16)$ \\
Oceania & $(39,25)$ & $(42,27)$ & $(31,19)$ & $(34,12)$ & $(33,27)$ & $(33,27)$ \\
South America & $(27,14)$ & $(23,15)$ & $(16,12)$ & $(26,15)$ & $(46,28)$ & $(35,24)$ \\
Central America & $(29,17)$ & $(29,19)$ & $(26,17)$ & $(29,25)$ & $(47,24)$ & $(46,27)$ \\
Caribbean & $(24,20)$ & $(24,20)$ & $(24,11)$ & $(18,12)$ & $(24,12)$ & $(46,26)$ \\
France & $(15,13)$ & $(30,14)$ & $(25,12)$ & $(43,31)$ & $(23,20)$ & $(40,21)$ \\
Germany & $(32,10)$ & $(25,8)$ & $(25,9)$ & $(32,15)$ & $(24,12)$ & $(24,12)$ \\
Italy & $(44,15)$ & $(34,15)$ & $(34,15)$ & $(57,27)$ & $(18,14)$ & $(30,23)$ \\
Netherlands & $(36,14)$ & $(37,14)$ & $(26,19)$ & $(26,19)$ & $(22,10)$ & $(32,11)$ \\
Spain & $(28,8)$ & $(12,6)$ & $(24,8)$ & $(14,9)$ & $(14,3)$ & $(14,9)$ \\
Sweden & $(39,11)$ & $(39,11)$ & $(39,11)$ & $(38,15)$ & $(23,20)$ & $(24,15)$ \\
Switzerland & $(15,12)$ & $(44,38)$ & $(16,13)$ & $(31,21)$ & $(26,17)$ & $(26,17)$ \\
UK & $(24,14)$ & $(22,14)$ & $(32,24)$ & $(51,38)$ & $(41,34)$ & $(47,14)$ \\
Japan & $(31,25)$ & $(28,9)$ & $(47,19)$ & $(23,21)$ & $(47,34)$ & $(39,9)$ \\
South Korea & $(32,18)$ & $(27,17)$ & $(28,25)$ & $(31,21)$ & $(39,15)$ & $(50,36)$ \\
PRC and Hongkong & $(40,18)$ & $(16,13)$ & $(41,21)$ & $(25,15)$ & $(50,21)$ & $(42,34)$ \\
ROC & $(40,21)$ & $(40,31)$ & $(37,33)$ & $(37,33)$ & $(37,16)$ & $(37,16)$ \\
Australia & $(55,19)$ & $(37,21)$ & $(37,12)$ & $(36,12)$ & $(49,33)$ & $(48,33)$ \\
Argentina & $(23,15)$ & $(30,26)$ & $(15,13)$ & $(17,15)$ & $(41,39)$ & $(41,40)$ \\
Brazil & $(26,15)$ & $(14,11)$ & $(46,12)$ & $(39,24)$ & $(39,22)$ & $(50,12)$ \\
Colombia & $(29,15)$ & $(29,16)$ & $(39,11)$ & $(36,11)$ & $(27,23)$ & $(19,10)$ \\
Venezuela & $(30,15)$ & $(28,15)$ & $(26,15)$ & $(18,15)$ & $(48,15)$ & $(37,17)$ \\
\hline
\end{tabular}

Table 9 reports the results for out-of-sample forecasting of U.S. Tourist arrivals by country of origin. We can infer from the RRMSE criterion that, SSA outperforms ARIMA at forecasting U.S. Tourist arrivals at all horizons for all countries of origin with the exception of Mexico at $h$ $=3$ steps ahead. Furthermore, it is clear from the results that on average, SSA is $53 \%, 49 \%$, $44 \%, 47 \%, 46 \%$ and $41 \%$ better than ARIMA at horizons of $h=1,3,6,12,24$ and 36 months ahead respectively for forecasting U.S. Tourist arrivals by individual country of origin. These results prove that by employing SSA to analyse and forecast the monthly U.S. Tourists arrivals data by country of origin we can obtain significantly more accurate forecasts than those possible with the optimal ARIMA for both short and long term fluctuations in tourist arrivals into the U.S. from each country. We test the results further for statistical significance. Accordingly, we find that except for tourist arrivals from Mexico, every other forecasting result obtained in this study is statistically significant. This suggests that when forecasting tourist arrivals from Mexico there is no difference between the forecasting accuracy of the ARIMA and SSA models. 
Table 9: Forecasting results for U.S. Tourist arrivals by country of origin.

\begin{tabular}{|c|c|c|c|c|c|c|}
\hline \multirow[b]{2}{*}{ Origin } & \multicolumn{4}{|c|}{$\frac{S S A}{A R I M A}$} & \multirow[b]{2}{*}{24} & \multirow[b]{2}{*}{36} \\
\hline & 1 & 3 & 6 & 12 & & \\
\hline Canada & $0.27^{*}$ & $0.32^{*}$ & $0.40^{*}$ & $0.37^{*}$ & $0.30 *$ & $0.36^{*}$ \\
\hline Mexico & 0.98 & 0.96 & 1.07 & 0.99 & 0.93 & $0.77^{* *}$ \\
\hline Total Overseas & $0.44^{*}$ & $0.48^{*}$ & $0.42^{*}$ & $0.48^{*}$ & $0.33^{*}$ & $0.43^{*}$ \\
\hline Western Europe & $0.46^{*}$ & $0.50^{*}$ & $0.53^{*}$ & $0.47^{*}$ & $0.43^{*}$ & $0.44^{*}$ \\
\hline Eastern Europe & $0.34^{*}$ & $0.37^{*}$ & $0.42^{*}$ & $0.41^{*}$ & $0.42^{*}$ & $0.39^{*}$ \\
\hline Asia & $0.54^{*}$ & $0.68^{*}$ & 0.72 & 0.91 & $0.80 *$ & 0.91 \\
\hline Middle East & $0.55^{*}$ & $0.42^{*}$ & $0.47^{*}$ & $0.37^{*}$ & $0.38 *$ & $0.46^{*}$ \\
\hline Africa & $0.28^{*}$ & $0.39^{*}$ & $0.45^{*}$ & $0.36^{*}$ & $0.26^{*}$ & $0.24^{*}$ \\
\hline Oceania & $0.40^{*}$ & $0.43^{*}$ & $0.51^{*}$ & $0.53^{*}$ & $0.60 *$ & $0.75^{* *}$ \\
\hline South America & $0.43^{*}$ & $0.45^{*}$ & $0.56^{*}$ & $0.50 *$ & $0.49^{*}$ & 0.82 \\
\hline Central America & $0.44^{*}$ & $0.45^{*}$ & $0.52^{*}$ & $0.46^{*}$ & $0.34^{*}$ & $0.46^{*}$ \\
\hline Caribbean & $0.34^{*}$ & $0.38^{*}$ & $0.43^{*}$ & $0.34^{*}$ & $0.49 *$ & $0.61^{*}$ \\
\hline France & $0.36^{*}$ & $0.45^{*}$ & $0.42^{*}$ & $0.36^{*}$ & $0.52^{*}$ & $0.42^{*}$ \\
\hline Germany & $0.60^{*}$ & $0.51^{*}$ & $0.64^{*}$ & $0.61^{*}$ & $0.64^{*}$ & $0.60^{*}$ \\
\hline Italy & $0.31^{*}$ & $0.37^{*}$ & $0.41^{*}$ & $0.38^{*}$ & $0.35^{*}$ & $0.44^{*}$ \\
\hline Netherlands & $0.48^{*}$ & $0.53^{*}$ & $0.47^{*}$ & $0.44^{*}$ & $0.44^{*}$ & $0.43^{*}$ \\
\hline Spain & $0.60^{*}$ & $0.78^{*}$ & $0.76^{* *}$ & $0.62^{*}$ & $0.65^{*}$ & 0.93 \\
\hline Sweden & $0.53^{*}$ & $0.62^{*}$ & $0.72^{*}$ & $0.69^{*}$ & $0.62^{*}$ & $0.47^{*}$ \\
\hline Switzerland & $0.48^{*}$ & $0.50^{*}$ & $0.54^{*}$ & $0.50 *$ & $0.48^{*}$ & $0.42^{*}$ \\
\hline United Kingdom & $0.52^{*}$ & $0.49^{*}$ & $0.61^{*}$ & $0.65^{*}$ & $0.72^{* *}$ & 0.92 \\
\hline Japan & $0.62^{*}$ & $0.83^{*}$ & 0.82 & $0.71^{* *}$ & $0.66^{*}$ & 0.96 \\
\hline South Korea & $0.48^{*}$ & $0.49^{*}$ & $0.73^{*}$ & $0.79^{*}$ & 0.88 & 0.91 \\
\hline PRC \& Hongkong & $0.51^{*}$ & $0.52^{*}$ & $0.56^{*}$ & $0.47^{*}$ & $0.73^{* *}$ & $0.64^{*}$ \\
\hline ROC (Taiwan) & $0.50^{*}$ & $0.44^{*}$ & $0.48^{*}$ & $0.58^{*}$ & $0.50^{*}$ & $0.40^{*}$ \\
\hline Australia & $0.44^{*}$ & $0.45^{*}$ & $0.48^{*}$ & $0.49^{*}$ & $0.61^{*}$ & $0.59^{*}$ \\
\hline Argentina & $0.54^{*}$ & $0.62^{*}$ & $0.75^{*}$ & $0.64^{*}$ & $0.61^{*}$ & $0.59^{*}$ \\
\hline Brazil & $0.53^{*}$ & $0.53^{*}$ & $0.58^{*}$ & $0.53^{*}$ & $0.49 *$ & $0.49^{*}$ \\
\hline Colombia & $0.34^{*}$ & $0.38^{*}$ & $0.41^{*}$ & $0.41^{*}$ & $0.35^{*}$ & $0.66^{*}$ \\
\hline Venezuela & $0.42^{*}$ & $0.34^{*}$ & $0.34^{*}$ & $0.44^{*}$ & $0.53^{*}$ & $0.52^{*}$ \\
\hline Average & 0.47 & 0.51 & 0.56 & 0.53 & 0.54 & 0.59 \\
\hline
\end{tabular}

Interestingly, when forecasting U.S. Tourist arrivals from Mexico, the optimal SSA choice for the number of eigenvalues, $r$ is $r=1$ at horizons of 1,3 and 6 steps ahead. This in turn means that the SSA model is relying on the trend alone to forecast future data points for Mexico. As such we find it important to briefly comment on this fact. For this purpose, in Figure 4 we have selected the time series for Mexico and three other time series which were shown to have structural breaks (see, Table 2). Upon closer analysis it is clear that whilst all four time series shown here are affected by structural breaks, the time series for Mexico shows signs of a major structural break shifting U.S. tourist arrivals from Mexico starting December 2009. The magnitude of this break has implications on SSA's modelling capabilities especially as we do not incorporate change point detection methods in this paper. This particular time series alone provides some useful topics for future research as it suggests that SSA change point detection should be incorporated into the tourist arrivals forecasting models so that the technique is equipped to provide improved forecasts in the face of similar time series. In line with ensuring equality between the other forecasting models adopted in this study, we have used the most 

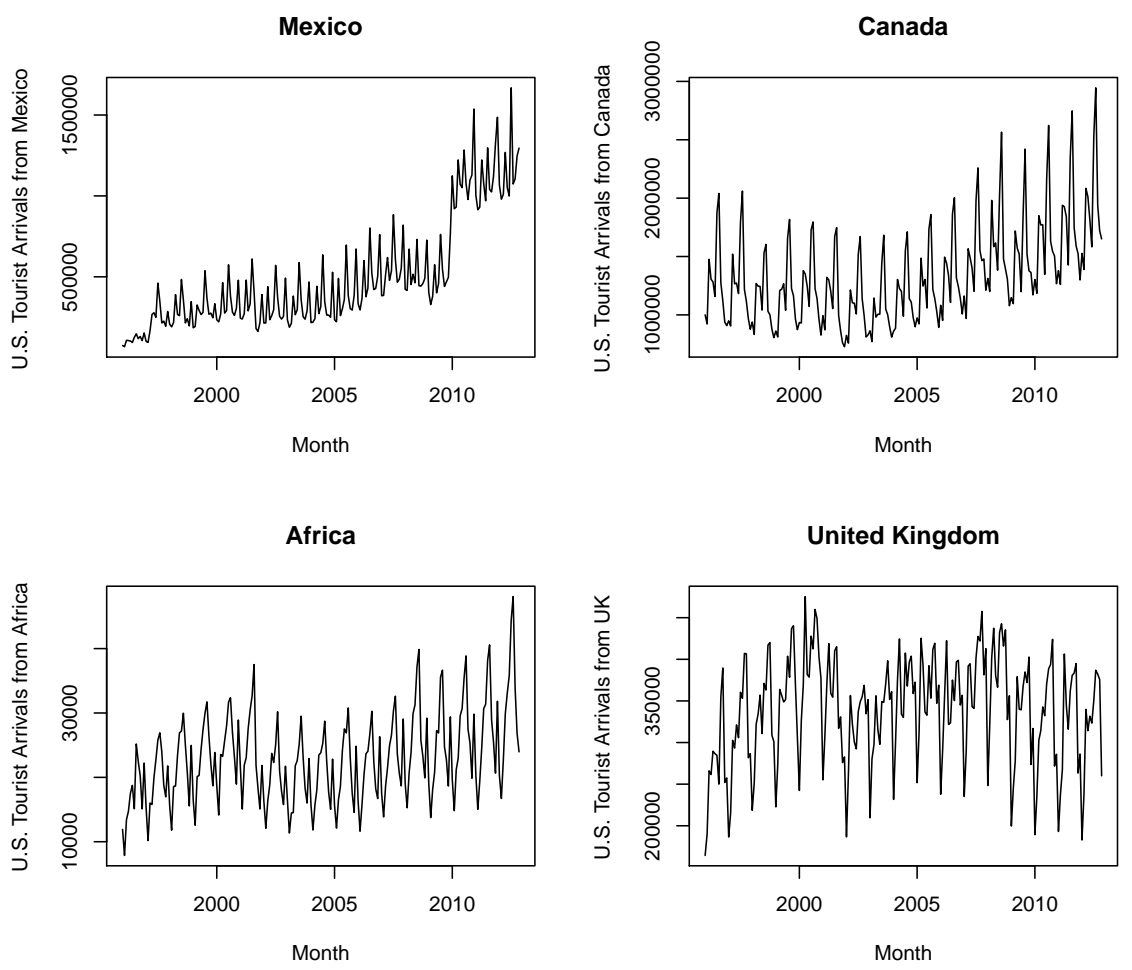

Figure 4: Selected U.S. Tourist arrivals time series (Jan. 1996 - Nov. 2012).

\section{Conclusion}

The starting point of this paper, as with many other authors, was the importance of accurate forecasts of tourism demand to investors, managers and policy makers. The existence of a high degree of volatility in tourism demand not only increases this need but creates a need for forecasting techniques that cope well with this volatility.We introduce Singular Spectrum Analysis (SSA) as a new model for forecasting inbound U.S. Tourist arrivals. The U.S. Tourist arrivals time series are analyzed in total and by country of origin.

Our analysis compared the forecasting accuracy of the newly proposed technique, the Vector SSA model, with the forecasting accuracy of the several different widely used forecasting models. These most were the accurate version of ARIMA, known as Automatic-ARIMA, an Exponential Smoothing model known as ETS and a feed-forward Neural Network model known as nnetar. Automatic-ARIMA, ETS and nnetar are all provided as automatic forecasting techniques within the forecast package within the $\mathrm{R}$ software. We found that the newly proposed U.S. Tourist arrivals forecasting model of SSA outperforms all three of these models (ARIMA, ETS and Neural Networks). The $w$-correlations (Table 4) also provide an explanation for one reason behind the outstanding performance recorded by the VSSA model as they indicate clearly that the VSSA forecasting algorithm is highly successful in separating the signal from the noise found in the U.S. Tourist arrivals series.

Moreover, this paper further uncovers substantial evidence to support the discontinuation of the use of ARIMA, ETS and feed-forward Neural Networks as a model for forecasting inbound 
U.S. inbound tourist arrivals in the future, based on the RMSE, RRMSE and DC criteria along with statistically significant results. The results also show that the basic Vector-SSA model with the optimal decomposition is able to outperform the optimal ARIMA or, Automatic-ARIMA model, ETS and nnetar of Hyndman and Khandakar (2008) in forecasting U.S. Tourist arrivals. Our results indicate that the nonparametric SSA model is on average $49 \%$ more accurate than the parametric model of ARIMA, 58\% more accurate than ETS, and $69 \%$ more accurate than the feed-forward Neural Network model (nnetar) at forecasting tourist arrivals. This provides sound evidence for National Statistical Agencies in U.S. and elsewhere to consider introducing SSA as a more reliable method of forecasting tourist arrivals. Furthermore, the SSA technique has been shown here to provide not only the most accurate forecasts based on the lowest RMSE values, but also with statistically significant results in comparison to the rest of the models.

This paper contributes to the literature on forecasting tourism demand in several ways. Firstly, we show that the SSA technique can be used as a reliable demand forecasting technique for tourism in the future, using its application to inbound tourist arrivals in the U.S. as an example. In doing so, we also increase the number of options available for demand forecasting in tourism. Secondly, we show that SSA outperforms the optimal ARIMA model of Hyndman and Khandakar (2008). This is an important finding as ARIMA models are widely used in forecasting tourism demand at present. The results are statistically significant and provide strong evidence to support the discontinuation of ARIMA as a tourism demand forecasting technique for the U.S. at least. Given the introduction of SSA and its strong performance with U.S. data it would be interesting to see how well the model performs in forecasting tourism demand in other nations. Thirdly, we also evaluate the SSA technique against an exponential smoothing and neural network model which shows the basic Vector SSA to be superior. Whilst more research work should be conducted on the comparison of SSA especially against neural networks in the future, the initial evidence is supportive of the use of SSA.

Overall, given the importance of forecasting tourism demand and the important requirement that such forecasts be able to cope well with volatility in demand, this paper offers a new technique to forecasters in this area. The evidence from the U.S. data is that it offers the prospect of better forecasting accuracy than the pick of those techniques previously employed. Improvements in forecasting accuracy should provide a basis for more efficient resource allocation by, in particular, investors and managers in tourism.

In terms of the implications of this paper for further research there are several. In this paper we compared the performance of SSA to three of the most important existing alternative techniques. It would be worthwhile extending this analysis in the future to a wider of alternative techniques. The encouraging results from employing SSA to forecast U.S. inbound tourism reported in this paper also suggests that it may be worthwhile in future research to build a multivariate SSA model to forecast tourist arrivals. Here, it would be interesting to evaluate the spatio-temporal correlations between tourist arrivals from various countries (as proposed in Sato, 2012) so that this information could be used to enhance the multivariate SSA model to enable more accurate forecasts. Finally, the use of hybrid models has been common in the literature concerning the forecasting of tourism demand. It would be both interesting and of potential value for future research to consider how the SSA technique performs in a hybrid model. Moreover, the presence of structural breaks in U.S. Tourist arrivals suggests that it would also be interesting to evaluate the impact on the forecasts of replacing KPSS tests with the Bai-Perron (2003) test for determining the number of differences in the ARIMA models. The results from forecasting tourist arrivals from Mexico also makes it clear that future studies should consider incorporating SSA change point detection for forecasting U.S. Tourist arrivals. Finally, it is possible that different categories of tourism may be behaviourally different in a way 
678 that is relevant for other forecasting uses. In this paper, owing to data limitations we have not 679 been successful in analysing U.S. Tourist arrivals based on purpose of visit and future research 680 could benefit immensely if such data was made available by the relevant authorities. 


\section{References}

Alvarez-Diaz, M., \& Rossello-Nadal, J. (2010). Forecasting British tourist arrivals in the Balearic Islands using meteorological variables. Tourism Economics, 16, 153-168.

Andrawis, R. R., Atiya, A. F., \& El-Shishiny, H. (2011). Combination of long term and short term forecasts, with application to tourism demand forecasting. International Journal of Forecasting, 27, 870-886.

Assaf, A. G., Barros, C. P., \& Gil-Alana, L. A. (2011). Persistence in the short and long-term tourist arrivals to Australia. Journal of Travel Research, 50, 213-22.

Athanasopoulos, G., \& de Silva, A. (2012). Multivariate exponential smoothing for forecasting tourist arrivals. Journal of Travel Research, 51, 640-652.

Bai J., and Perron P. (2003). Computation and Analysis of Multiple Structural Change Models. Journal of Applied Econometrics, 18, 1-22.

Beneki, C., Eeckels, B., and Leon, C. (2012). Signal extraction and forecasting of the UK tourism income time series: A Singular Spectrum Analysis approach. Journal of Forecasting, 31, 391-400.

Beneki, C., \& Silva, E. S. (2013). Analysing and forecasting European Union energy data. International Journal of Energy and Statistics, 1, 127-141.

Bollerslev, T. (1986). Generalized autoregressive conditional heteroskedasticity. Journal of econometrics, 31, 307-327.

Box, G., \& Jenkins, G. (1970). Time series analysis: Forecasting and control. San Francisco: Holden-Day.

Broomhead, D. S., \& King, G. P. (1986a). Extracting qualitative dynamics from experimental Data. Physica D: Nonlinear Phenomena, 20, 217-236.

Broomhead, D. S., \& King, G. P. (1986b). On the qualitative analysis of experimental dynamical systems. In Nonlinear Phenomena and Chaos. S., (ed.) Adam Hilger: Bristol, 113-144.

Cang, S. (2011). A non-linear tourism demand forecast combination model. Tourism Economics, 17, 5-20.

Chan, F., \& Lim, C. (2011). Spectral analysis of seasonality in tourism demand. Mathematics and Computers in Simulation, 81, 1409-1418.

Cho, V. (2003). A comparison of three different approaches to tourist arrival forecasting. Tourism Management, 24, 323-330.

Chu, F. L. (2008). A fractionally integrated autoregressive moving average approach to forecasting tourism demand. Tourism Management, 29, 79-88.

Chu, F. L. (2011). A piecewise linear approach to modeling and forecasting demand for Macau tourism. Tourism Management, 32, 1414-1420.

Coshall, J. T. (2009). Combining volatility and smoothing forecasts of UK demand for international tourism. Tourism Management, 30, 495-511. 
Coshall, J. T., \& Charlesworth, R. (2011). A management orientated approach to combination forecasting of tourism demand. Tourism Management, 32, 759-769.

Croes, R. R., \& Vanegas Sr, M. (2005). An econometric study of tourist arrivals in Aruba and its implications. Tourism Management, 26, 879-890.

Fourie, J., \& Santana-Gallego, M. (2011). The impact of mega-sport events on tourist arrivals. Tourism Management, 32, 1364-1370.

Ghil, M., Allen, R. M., Dettinger, M. D., Ide, K., Kondrashov, D., Mann, M. E., Robertson, A., Saunders, A., Tian, Y., Varadi, F., \& Yiou, P. (2002). Advanced spectral methods for climatic time series. Reviews of Geophysics, 40, 3.1-3.4.1.

Ghodsi, M., Hassani, H., Sanei, S., \& Hicks, Y. (2009). The use of noise information for detection of temporomandibular disorder. Biomedical Signal Processing and Control, 4, 79-85.

Gil-Alana, L. A. (2005). Modelling international monthly arrivals using seasonal univariate long-memory processes. Tourism Management, 26, 867-878.

Goh, C., \& Law, R. (2002). Modeling and forecasting tourism demand for arrivals with stochastic nonstationary seasonality and intervention. Tourism Management, 23, 499-510.

Golyandina, N., Nekrutkin, V., \& Zhigljavsky, A. (2001). Analysis of time series structure: SSA and related techniques. Chapman \& Hall/CRC.

Gounopoulos, D., Petmezas, D., \& Santamaria, D. (2012). Forecasting tourist arrivals in Greece and the impact of macroeconomic shocks from the countries of tourists origin. Annals of Tourism Research, 39, 641-666.

Greenidge, K. (2001). Forecasting tourism demand: An STM approach. Annals of Tourism Research, 28, 98-112.

Hadavandi, E., Ghanbari, A., Shahanaghi, K., \& Abbasian-Naghneh, S. (2011). Tourist arrival forecasting by evolutionary fuzzy systems. Tourism Management, 32, 1196-1203.

Harvey, D. I., Leybourne, S. J., \& Newbold, P. (1997). Testing the equality of prediction mean squared errors. International Journal of Forecasting, 13, 281-291.

Hassani, H. (2007). Singular Spectrum Analysis (SSA): Methodology and comparison. Journal of Data Science, 5, 239-257.

Hassani, H., Heravi, H., \& Zhigljavsky, A. (2009). Forecasting European industrial production with Singular Spectrum Analysis. International Journal of Forecasting, 25, 103-118.

Hassani, H., Heravi, H., \& Zhigljavsky, A. (2012). Forecasting UK industrial production with multivariate singular spectrum analysis. Journal of Forecasting, 32, 395-408.

Hassani, H., \& Mahmoudvand, R. (2013). Multivariate Singular Spectrum Analysis: A general view and new vector forecasting approach. International Journal of Energy Statistics, 1, 55-83.

Hassani, H., Soofi, A. S., \& Zhigljavsky, A. (2013). Predicting inflation dynamics with singular spectrum analysis. Journal of the Royal Statistical Society A, 176, 743-760.

Hassani, H., \& Thomakos, D. (2010). A review on Singular Spectrum Analysis for economic and financial time series. Statistics and its Interface, 3, 377-397. 
Hui, T. K., \& Yuen, C. C. (2002). A study in the seasonal variation of Japanese tourist arrivals in Singapore. Tourism Management, 23, 127-131.

Hyndman, R. J. (2014). Unit root tests and ARIMA models. Available via: http://robjhyndman.com/hyndsight/unit-root-tests/

Hyndman, R. J. \& Athanasopoulos G. (2013). Forecasting: principles and practice, OTexts: Australia. OTexts.com/fpp.

Hyndman, R. J., Athanasopoulos G., Razbash, S., Schmidt, D., Zhou, Z., Khan, Y., \& Bergmeir, C. (2013). Package forecast: Forecasting functions for time series and linear models. Available via: http://cran.r-project.org/web/packages/forecast/forecast.pdf

Hyndman, R. J., \& Khandakar, Y. (2008). Automatic time series forecasting: The forecast Package for R. Journal of Statistical Software, 27, 1-22.

Hyndman, R. J., Koehler, A. B., Snyder, R. D., \& Grose, S. (2002). A state space framework for automatic forecasting using exponential smoothing methods. International Journal of Forecasting, 18, 439-454.

Jackman, M., \& Greenidge, K. (2010). Modelling and forecasting tourist flows to Barbados using structural time series models. Tourism and Hospitality Research, 10, 1-13.

Kim, S. S., \& Wong, K. K. (2006). Effects of news shock on inbound tourist demand volatility in Korea. Journal of Travel Research, 44, 457-466.

Kim, J. H., Wong, K., Athanasopoulos, G., \& Liu, S. (2011). Beyond point forecasting: Evaluation of alternative prediction intervals for tourist arrivals. International Journal of Forecasting, 27, 887-901.

Kwiatkowski, D., Phillips, P. C., Schmidt, P., \& Shin, Y. (1992). Testing the null hypothesis of stationarity against the alternative of a unit root. Journal of Econometrics, 54, 159-178.

Lim, C., \& McAleer, M. (2001). Forecasting tourist arrivals. Annals of Tourism Research, 28, 965-977.

Lisi, F., \& Medio, A. (1997). Is a random walk the best exchange rate predictor? International Journal of Forecasting, 13, 255-267.

Makridakis, S. G., Wheelwright, S. C., \& Hyndman R. J. (1998). Forecasting: Methods and applications. 3rd ed., Wiley: NY.

Mao, C. K., Ding, C. G., \& Lee, H. Y. (2010). Post-SARS tourist arrival recovery patterns: An analysis based on a catastrophe theory. Tourism Management, 31, 855-861.

Naud, W. A., \& Saayman, A. (2005). Determinants of tourist arrivals in Africa: a panel data regression analysis. Tourism Economics, 11, 365-391.

Nelson, L. A., Dickey, D. A., \& Smith, J. M. (2011). Estimating time series and cross section tourism demand models: Mainland United States to Hawaii data. Tourism Management, 32, $28-38$.

Page, S., Song, H., \& Wu, D. C. (2012). Assessing the impacts of the global economic crisis and swine flu on inbound tourism demand in the United Kingdom. Journal of Travel Research, $51,142-153$. 
Pepelyshev, A. (2010). Comparison of Recurrent and Vector forecasting. UK-China workshop on Singular Spectrum Analysis and its applications, Cardiff, September 20, 2010. Available via http://ssa.cf.ac.uk/pepelyshev/pepelyshev_ssa_forecast.pdf.

Sato, A-H. (2012). Impact of the Great East Japan earthquake on hotel industry in Pacific Tohoku prefectures. Progress of Theoretical Physics Supplement, 194, 165-172.

Seetanah, B., Durbarry, R., \& Ragodoo, J. F. (2010). Using the panel cointegration approach to analyse the determinants of tourism demand in South Africa. Tourism Economics, 16, 715-729.

Seetaram, N. (2010). Use of dynamic panel cointegration approach to model international arrivals to Australia. Journal of Travel Research, 49, 414-422.

Shareef, R., \& McAleer, M. (2007). Modelling the uncertainty in monthly international tourist arrivals to the Maldives. Tourism Management, 28, 23-45.

Shen, S., Li, G., \& Song, H. (2008). An assessment of combining tourism demand forecasts over different time horizons. Journal of Travel Research, 47, 197-207.

Shen, S., Li, G., \& Song, H. (2011). Combination forecasts of international tourism demand. Annals of Tourism Research, 38, 72-89.

Silva, E. S. (2013). A combination forecast for energy related $\mathrm{CO}_{2}$ emissions in the United States. International Journal of Energy and Statistics, 1, 269-279.

Smeral, E. (2010). Impacts of the world recession and economic crisis on tourism: Forecasts and potential risks. Journal of Travel Research, 49, 31-38.

Song, H., Dwyer, L., Li, G., \& Cao, Z. (2012). Tourism economics research: A review and assessment. Annals of Tourism Research, 39, 1653-1682.

Song, H., Gao, B. Z., \& Lin, V. S. (2013). Combining statistical and judgmental forecasts via a web-based tourism demand forecasting system. International Journal of Forecasting, 29, 295-310.

Song, H., \& Li, G. (2008). Tourism demand modelling and forecasting - a review of recent research. Tourism Management, 29, 203-220.

Song, H., Li, G., Witt, S. F., \& Fei, B. (2010). Tourism demand modelling and forecasting: How should demand be measured? Tourism Economics, 16, 63-81.

Song, H., Li, G., Witt, S. F., \& Athanasopoulos, G. (2011). Forecasting tourist arrivals using time-varying parameter structural time series models. International Journal of Forecasting, 27, 855-869.

Wan, S. K., Wang, S. H., \& Woo, C. K. (2013). Aggregate vs. disaggregate forecast: Case of Hong Kong. Annals of Tourism Research, 42, 434-438.

Zhang, G., Patuwo, B. E., \& Hu, M. Y. (1998). Forecasting with artificial neural networks: the state of the art. International Journal of Forecasting, 14, 35-62. 